\title{
The statistical multifragmentation model for liquid-gas phase transition with a compressible nuclear liquid
}

\author{
V. V. Sagun ${ }^{1}$, A. I. Ivanytskyi ${ }^{1}$, K. A. Bugaev ${ }^{1,2}$ and I. N. Mishustin ${ }^{2,3}$ \\ ${ }^{1}$ Bogolyubov Institute for Theoretical Physics of the National Academy of Sciences of Ukraine, \\ Metrologichna str. $14^{b}$, Kiev-03680, Ukraine \\ ${ }^{2}$ Frankfurt Institute for Advanced Studies (FIAS), Goethe-University, \\ Ruth-Moufang Str. 1, 60438 Frankfurt upon Main, Germany and \\ 3 Kurchatov Institute, Russian Research Center, \\ Akademika Kurchatova Sqr., Moscow, 123182, Russia
}

We propose a new formulation of the statistical multifragmentation model based on the analysis of the virial expansion for a system of the nuclear fragments of all sizes. The developed model not only enables us to account for short-range repulsion, but also to calculate the surface free energy which is induced by the interaction between the fragments. We propose a new parameterization for the liquid phase pressure which allows us to introduce a compressible nuclear liquid into the statistical multifragmentation model. The resulting model is exactly solvable and has no irregular behavior of the isotherms in the mixed phase region that is typical for mean-field models. The general conditions for the 1-st and 2-nd (or higher) order phase transitions are formulated. It is shown that all endpoints of the present model phase diagram are the tricritical points, if the Fisher exponent $\tau$ is in the range $\frac{3}{2} \leq \tau \leq 2$. The treatment of nuclear liquid compressibility allows us to reduce the tricritical endpoint density of the statistical multifragmentation model to one third of the normal nuclear density. A specific attention is paid to of the fragment size distributions in the region of a negative surface tension at supercritical temperatures.

Key words: Statistical multifragmentation model, Van der Waals extrapolation, surface tension, compressible nuclear liquid

PACS: $25.75 . \mathrm{Nq}, 25.75 .-\mathrm{q}$

\section{INTRODUCTION}

More than 30 years the statistical multifragmentation model (SMM) [1] is playing the leading role in studies of the nuclear multifragmentation reactions [2, 3] which, probably, is one of the most spectacular phenomena that is available for exploration in nuclear reactions at intermediate energies. Additionally, the SMM greatly stimulated the studies of phase transition (PT) in finite systems [25] and investigation of nonhomogeneous phases of strongly interacting matter in astrophysics [6] 8 and heavy-ion collisions [911].

A simplified version of the SMM without the Coulomb and asymmetry terms was proposed in [12, 13]. Its analytical solution was obtained in [14, 15], where an additional parameter, the Fisher exponent $\tau$, was introduced in the model. The value of Fisher exponent extracted from nuclear experiments [16, 17] turned out to be $\tau \simeq 1.8-1.9$, i.e. well below the prediction of the Fisher droplet model $(\mathrm{FDM}) \tau_{F} \simeq 2.209$ [18, 19, but in a good correspondence with the critical exponent analysis made in [20] for the simplified SMM. This fact initiated new attempts to analyze the multifragmentation data in order to extract the values of the exponent $\tau$ and critical temperature [21 23]. These studies gave a first evidence that the nuclear liquid-gas PT has the tricritical endpoint rather than the critical one [20]. 
The successes achieved by the SMM in describing many sets of different experimental data gave enough proofs of its validity and this very fact prevented researches from asking two simple conceptual questions: why does the SMM perfectly work in describing the low density nuclear vapour that appears after the multifragmentation, and why the SMM is unable to describe the high densities of nuclear matter achieved, for example, in heavy ion collisions. At first glance the answer on the second question seems to be very trivial: the SMM considers nucleons and all nuclear fragments as incompressible objects. This can be seen from the excluded volume of the $k$-nucleon fragment which is $V_{k}=\frac{k}{\rho_{0}}$, where $\rho_{0} \simeq 0.16 \mathrm{fm}^{3}$ denotes the normal nuclear density. Thus, the limiting particle density appears in the SMM due to the Van der Waals like treatment of the short range repulsion between the fragments. However, in this case we really face a difficult problem to get the answer on the first of the above questions and to explain the reason why the SMM is so good in describing the experimental data at freeze-out density which is between $\frac{1}{12}$ and $\frac{1}{6}$ of $\rho_{0}$. It is well known that to describe the thermodynamics at low densities one has to use the virial expansion and account, at least, for the second virial coefficients $b_{j k}=\frac{2}{3} \pi\left(R_{j}+R_{k}\right)^{3}$ between all pairs of fragments of the hard core radii $R_{j}$ and $R_{k}$ [24 27]. The real problem, however, is that the SMM employs not the second virial coefficients which provide the description of low density matter, but uses the proper volumes of the $k$-nucleon fragments $V_{k}=\frac{4}{3} \pi R_{k}^{3}$, which, usually, enter in the high density limit [28, 29]! Therefore, in order to understand why the SMM is successful at low densities, first of all, we have to return to its basic assumptions and find out, how the virial coefficients appear in this model.

The simplified version of the SMM [12, 13] which is solved analytically in [14, 15] is much more elaborate than the FDM [18] since, in contrast to the latter one, the SMM explicitly contains the nonzero proper sizes of all fragments and, hence, the liquid phase. However, in the standard SMM the nuclear liquid is incompressible, that is too rough approximation at higher temperatures. Moreover, the critical and tricritical endpoints of the simplified SMM [14, 20] appear at the density of a liquid phase $\rho=\rho_{l}(T=0)=\rho_{0}$, while in ordinary substances the critical density is about one third of that one for low temperature liquid phase [24, 30, 31. In present paper we extend the SMM to account for the compressibility of nuclear liquid and show how the equation for the surface tension coefficient induced by interaction between the nuclear fragments naturally appears from the virial expansion. Such an analysis allows us to answer the two conceptual questions formulated above. The developed approach has common features with Refs. [18, 30, 31, but compared to these works we obtain an equation for the surface tension coefficient which is induced by interaction between the nuclear fragments. Therefore, the developed model can be considered as a further refinement of the ideas formulated in [18, 30, 31].

The work is organized as follows. In Section 2 we give a heuristic derivation of the equations for the gaseous phase pressure and for the surface tension induced by the hard core repulsion. The conditions of the 1-st and 2-nd order PT of a liquid-gas type are formulated in Section 3 . The properties of the liquid phase pressure and the phase diagrams for various parameterizations of the present model are discussed in Section 4. Section 5 is devoted to an analysis of the fragment size distributions when going from subcritical to supercritical temperatures. The conclusions are formulated in Section 6.

\section{SMM WITH HARD CORE REPULSION}

In order to recapitulate the formal steps of obtaining the Van der Waals EoS in the grand canonical ensemble, let us consider the one-component gas with the hard core repulsion. The pressure of such a gas with the temperature $T$ and chemical potential $\mu$ in the nonrelativistic approximation is given by

$$
p=T \phi(T) \exp \left[\frac{\mu-a p}{T}\right], \quad \text { with } \quad \phi(T)=g \int \frac{d^{3} \mathbf{k}}{(2 \pi)^{3}} \exp \left[-\frac{\mathbf{k}^{2}}{2 m T}\right] .
$$


Here $a$ denotes the second virial coefficient, $\phi(T)$ is a thermal density of particles having the mass $m$ (below this will be the nucleon mass $m=940 \mathrm{MeV}$ ) and the degeneracy factor $g$. For low densities Eq. (1) can be obtained from the virial expansion [24] at low densities

$$
p \simeq T \phi e^{\frac{\mu}{T}}\left(1-a \phi e^{\frac{\mu}{T}}\right)
$$

in the following sequence of steps: first, one approximates the particle density as $\phi e^{\frac{\mu}{T}} \simeq \frac{p}{T}$, using the fact that at low densities such approximation is obeyed; second, now the obtained term is further approximated as $1-a \frac{p}{T} \simeq \exp \left[-a \frac{p}{T}\right]$. As a result Eq. (1) is reproduced. The final step is to extrapolate Eq. (1) to high densities. In fact, the above result can be obtained from the traditional Van der Waals EoS without attraction either by its direct integration [25] or by the maximum term method [26].

Let us apply the same steps to the system of $N$-sorts of particles of the hard core radii $R_{k}$, with $k=1,2, \ldots, N$. Then the virial expansion of the gas pressure up to second order in particle density is given by

$$
p=T \sum_{k=1}^{N} \phi_{k} e^{\frac{\mu_{k}}{T}}\left(1-\sum_{n=1}^{N} a_{k n} \phi_{n} e^{\frac{\mu_{n}}{T}}\right)
$$

where $\phi_{n}(T)=g_{n} \int \frac{d^{3} \mathbf{k}}{(2 \pi)^{3}} \exp \left[-\frac{\mathbf{k}^{2}}{2 m_{n} T}\right]$ denotes the thermal density of particles of the degeneracy $g_{n}$ and mass $m_{n}$, and $a_{k n}$ is the second virial coefficients of particles $k$ and $n$

$$
a_{k n}=\frac{2}{3} \pi\left(R_{k}+R_{n}\right)^{3}=\frac{2}{3} \pi\left(R_{k}^{3}+3 R_{k}^{2} R_{n}+3 R_{k} R_{n}^{2}+R_{n}^{3}\right) .
$$

Of course, one can straightforwardly repeat the same steps as before with the only modification that for the multicomponent system each sort of particles, say $n$, generates its own pressure $p_{n}$ which should replace in Eq. (3) the particle density as $\phi_{n} e^{\frac{\mu_{n}}{T}} \simeq \frac{p_{n}}{T}$. Then one obtains the system of equations for partial pressures

$$
p_{k}=T \phi_{k} \exp \left[\frac{\mu_{k}}{T}-\sum_{n=1}^{N} a_{k n} \frac{p_{n}}{T}\right] \text {. }
$$

Such a system is known as the Lorentz-Berthelot mixture [26] for which the total pressure is the sum of all partial ones $p=\sum_{k=1}^{N} p_{k}$. It is clear that, in contrast to the one-component Van der Waals EoS, the inclusion of many-nucleon fragments accounts, at least partly, for the attractive interaction between their constituents.

The procedure of the Van der Waals extrapolation is, however, not unique and one can analyze different possibilities [26, 27]. Note that an order of mathematical operations is important [27]. If, in contrast to above treatment, one first explicitly substitutes the second virial coefficients (4) into expression for pressure (3) first and regroups the powers of radius $R_{k}$ of a $k$-nucleon fragment, then one would find

$$
p=T \sum_{k=1}^{N} \phi_{k} e^{\frac{\mu_{k}}{T}}\left[1-\frac{4}{3} \pi R_{k}^{3} \cdot \sum_{n=1}^{N} \phi_{n} e^{\frac{\mu_{n}}{T}}-4 \pi R_{k}^{2} \cdot \frac{1}{2} \sum_{n=1}^{N} R_{n} \phi_{n} e^{\frac{\mu_{n}}{T}}-2 \pi R_{k} \cdot \sum_{n=1}^{N} R_{n}^{2} \phi_{n} e^{\frac{\mu_{n}}{T}}\right] .
$$

Now one can see that the right hand side (r.h.s.) of Eq. (6) contains the expansion in powers of radius $R_{k}$. If one makes the Van der Waals extrapolation of pressure (6) in the same way, as we discussed earlier, then one can formally get the same structure of the surface free energy which was suggested in [30]. Thus, Eq. (6) contains the surface and curvature terms, which are, respectively, proportional to $R_{k}^{2}$ and $R_{k}$ in the square brackets on its r.h.s. However, in contrast to [30, 31] and their followers, the r.h.s. of Eq. (6) contains the bulk term as well which is proportional to $R_{k}^{3}$ or to the $k$-nucleon fragment volume. Hence, all fragments in (6) are interacting with each other via 
a hard core repulsion. Now it is clear that, if, in addition, one introduces the attraction via the spherical potential well of finite depth to model the proximity type interaction between the nuclear fragments, then the only bulk term would not be modified, while the surface and curvature terms would get an additional negative contributions which will be studied elsewhere.

Below we do not consider the curvature term explicitly, but we account for it implicitly by doubling the induced surface free energy coefficient $\Sigma$ and introducing

$$
\begin{aligned}
p & =T \sum_{k=1}^{N} \phi_{k} e^{\frac{\mu_{k}}{T}}\left[1-\frac{4}{3} \pi R_{k}^{3} \cdot \frac{p}{T}-4 \pi R_{k}^{2} \cdot \frac{\Sigma}{T}\right] \\
& \simeq T \sum_{k=1}^{N} \phi_{k} \exp \left[\frac{\mu_{k}}{T}-\frac{4}{3} \pi R_{k}^{3} \cdot \frac{p}{T}-4 \pi R_{k}^{2} \cdot \frac{\Sigma}{T}\right] .
\end{aligned}
$$

In order to guarantee a consistency with the derivation above, we assume that the induced surface free energy coefficient $\Sigma$ obeys the following equation

$$
\alpha \frac{\Sigma}{T} \simeq \alpha \sum_{k=1}^{N} R_{k} \phi_{k} \exp \left[\frac{\mu_{k}}{T}-\frac{4}{3} \pi R_{k}^{3} \cdot \frac{p}{T}-4 \pi R_{k}^{2} \cdot \alpha \frac{\Sigma}{T}\right] .
$$

Here the constant $\alpha>0$ is introduced due to the freedom of the Van der Waals extrapolation to high densities. In this way the present model accounts for higher order corrections compared to the low density virial expansion. As will be shown later, such a correction plays an important role at the vicinity of the tricritical endpoint. Also it is important to note that in (8) the induced surface free energy coefficient is extrapolated to high densities using the same ensemble of one-particle distribution functions $\phi_{k}$ for $k$-nucleon fragments as the one employed in (7). Now it is clear that at low particle densities Eqs. (7) and (8) correctly account for the virial expansion up to the second order while the deviation appears at the third virial coefficient.

To connect the above formula for pressure (7) with the gaseous phase pressure of the SMM, we parameterize the one-particle thermal densities of all $k$-nucleon fragments as

$$
\phi_{1}=z_{1}\left[\frac{m T}{2 \pi}\right]^{\frac{3}{2}} \exp \left[-\frac{\sigma_{0}(T)}{T}\right], \quad \phi_{k \geq 2}=g\left[\frac{m T}{2 \pi}\right]^{\frac{3}{2}} \frac{1}{k^{\tau}} \exp \left[\frac{\left(k p_{L} V_{1}-\mu_{k}\right)}{T}-\frac{\sigma_{0}(T)}{T} k^{\varkappa}\right],
$$

where $z_{1}=4$ is the degeneracy factor of nucleons, while the degeneracy factor for other fragments $g$ is, for simplicity, chosen to be 1 . In the final step of our heuristic derivation of the system of Eqs. (7) and (8) we presented the $k$-nucleon $(k>1)$ volume as $V_{k}=\frac{4}{3} \pi R_{k}^{3}=V_{1} k$, where $V_{1}=\frac{1}{\rho_{0}}$. The binding energy per nucleon of such a fragment is expressed via the free energy of liquid drop $-k V_{1} p_{L}(T, \mu)$ of same size and the corresponding chemical potential $\mu_{k}$. In the standard SMM the pressure of liquid phase is $p_{L}(T, \mu)=\frac{\mu+W(T)}{V_{1}}$ [14] and the $k$-nucleon fragment chemical potential $\mu_{k}=k \mu$ is expressed via the chemical potential of nucleon $\mu$. Obviously, for the standard SMM one finds the usual result for binding free energy $k p_{L} V_{1}-\mu_{k}=k W(T)$. In Eq. (9) the power $\tau$ is the Fisher topological exponent [18, which was introduced into the SMM in [14]. In actual simulation we use the value $\tau=1.9$ which is motivated by the experimental data [16, 17] (see below).

It is necessary to remind that in the standard SMM the temperature dependent binding energy is $W(T)=W_{0}+W_{F m}(T)$. Here $W_{0}=16 \mathrm{MeV}$ is the bulk binding energy per nucleon at vanishing temperature, while the term $W_{F m}(T) \equiv \frac{T^{2}}{\varepsilon_{0}}$ is the contribution of the excited states taken in the Fermi-gas approximation $\left(\varepsilon_{0}=16 \mathrm{MeV}\right)$. The r.h.s. of Eq. (9) contains the liquid phase pressure $p_{L}(T, \mu)$ of general form. Such a generalization of the SMM allows one to consider more complicated $\mu$ and $T$ dependencies of the liquid phase pressure than the one used in the original SMM. It is important that the resulting model automatically obeys the L. van Hove axioms of statistical mechanics [32], if in the liquid phase the liquid pressure obeys these axioms (see a discussion below). Due to this property the present model does not lead to an appearance of 
non-monotonic isotherms in the density-pressure plane which are typical for mean-field models of equation of state.

In Eq. (9) we have introduced the eigen part of the temperature-dependent surface tension $\sigma_{0}(T)$ for each fragment including the nucleons. This is a slight change compared to the standard SMM formulation [1, 14] which, however, does not strongly affect properties of the phase diagram, but somewhat simplifies the analysis of the model. The parameter $\varkappa$ is chosen to match that one of the SMM, i.e. $\varkappa=\frac{2}{3}$, but in the next section we analyze the properties of the PT diagram for a wider range of its values, namely for $\frac{2}{3} \leq \varkappa<1$.

Collecting all terms together, we rewrite Eqs. (7) and (8) as the following system

$$
\begin{aligned}
& \frac{p}{T}=\left[\frac{m T}{2 \pi}\right]^{\frac{3}{2}} \sum_{k=1}^{N} \frac{b_{k}(T)}{k^{\tau}} \exp \left[\frac{\left(p_{L}-p\right) V_{1}}{T} k-\frac{\left(\sigma_{1}+\sigma_{0}\right)}{T} k^{\varkappa}\right], \\
& \frac{\alpha \sigma_{1}}{3 T V_{1}}=\alpha\left[\frac{m T}{2 \pi}\right]^{\frac{3}{2}} \sum_{k=1}^{N} \frac{b_{k}(T)}{k^{\tau-\frac{1}{3}}} \exp \left[\frac{\left(p_{L}-p\right) V_{1}}{T} k-\frac{\left(\alpha \sigma_{1}+\sigma_{0}\right)}{T} k^{\varkappa}\right],
\end{aligned}
$$

for an infinite number of the sorts of nuclear fragments $N \rightarrow \infty$. Here the temperature dependent degeneracy is defined as $b_{1}(T) \equiv 4 \exp \left[\frac{-W(T)}{T}\right]$ and $b_{k>1}(T)=1$. In this way we account for the fact that, compared to larger fragments, the nucleons have no binding energy. One should bear in mind that for an infinite number of the sorts of nuclear fragments $N$ a redefinition of any finite number of internal partitions of $k$-nucleon fragments, i.e. $b_{k}(T)$, does not affect the divergency or convergency of the pressure (10) and the induced surface tension (11).

Choosing some temperature dependent parameterization for the eigen surface tension coefficient $\sigma_{0}(T)$ for the fragments, we obtain the closed system of equations to determine the pressure $p$ and the total surface tension coefficient $\sigma_{0}+\sigma_{1}$ for a given value of parameter $\alpha$. The pressure in this system is closely resembling the expression suggested in the simplified SMM to study the nuclear liquid-gas PT in thermodynamic limit [12, 14, 20].

The above consideration clearly demonstrates that the liquid drop parameterization of the internal free energy employed in the original SMM [1] is consistent with the Van der Waals extrapolation where the proper volume of fragments is used for the bulk part, while the rest of the hard core repulsion is accounted by the corresponding choice of the surface free energy. Similarly, the attractive interaction between the fragments is partially recorded in the surface free energy, while another part of attractive interaction is stored in the very fact that fragments with a certain binding energy are formed. By construction the EoS defined by (10) and (11) automatically reproduces the low density expansion. In addition, the suggested EoS contains the bulk part of free energy, which is applicable at high densities, and, consequently, such EoS may correctly reproduce properties of the liquid phase that is impossible in the model of non-interacting clusters [18, 30, 31].

\section{CONDITIONS FOR THE TRICRITICAL POINT EXISTENCE}

In order to study the nuclear liquid-gas PT Eqs. (10) and (11) should be supplemented by the EoS of the liquid phase pressure $p_{L}(T, \mu)$ to be substituted into an exact analytical expression of the simplified SMM found in [14, 20]. However, here we would like to follow a more traditional way for analyzing the necessary and sufficient conditions for the nuclear liquid-gas PT. For this purpose we rewrite (10) and 11 in terms of dimensionless variables $\xi_{1}, \xi_{2}$ and $\tilde{\xi}_{2}$

$$
\begin{aligned}
\xi_{1} & =-\xi_{L}+V_{1} I_{\tau}\left(\xi_{1}, \xi_{2}\right), \\
\xi_{2} & =\sigma_{0}+3 V_{1} I_{\tau-\frac{1}{3}}\left(\xi_{1}, \tilde{\xi}_{2}\right), \\
I_{\tau-q}\left(\xi_{1}, \xi_{2}\right) & \equiv\left[\frac{m T}{2 \pi}\right]^{\frac{3}{2}} \sum_{k=1}^{N} \frac{b_{k}(T)}{k^{\tau-q}} \exp \left[-\xi_{1} k-\xi_{2} k^{\varkappa}\right] .
\end{aligned}
$$


Here the dimensionless variables $\xi_{1}, \xi_{2}$ and $\tilde{\xi}_{2}$ are defined in terms of the bulk and surface components of the fragment's free energy taken per one nucleon as

$$
\begin{array}{ll}
\xi_{1} \equiv \frac{p V_{1}}{T}-\xi_{L}, & \xi_{L} \equiv \frac{p_{L} V_{1}}{T}, \\
\xi_{2} \equiv \frac{\sigma_{0}+\sigma_{1}}{T}, & \tilde{\xi}_{2} \equiv \frac{\sigma_{0}+\alpha \sigma_{1}}{T} .
\end{array}
$$

The variable $\xi_{1}$ is convenient for an analytical manipulations since in terms of this variable the gas and liquid coexistence condition $p(T, \mu)=p_{L}(T, \mu)$ reads as $\xi_{1}(T, \mu)=0$. Its solution $\mu=\mu_{c}(T)$ defines the phase diagram in the $T-\mu$ plane. Similarly, the variable $\xi_{2}$ which according to (13) describes the surface free energy coefficient as $T \xi_{2}$ is convenient to detect the critical (or tricritical) endpoint of the phase diagram. Indeed, below we explicitly demonstrate, that for $\xi_{2}\left(T, \mu_{c}(T)\right)>0$ the PT is of the 1-st order, whereas for $\xi_{2}\left(T, \mu_{c}(T)\right)=0$ the PT is of the 2-nd or higher order. The auxiliary variable $\tilde{\xi}_{2} \equiv \frac{\alpha \xi_{2}+(1-\alpha) \sigma_{0}}{T}$ is convenient both for analytical and numerical evaluations since instead of Eq. 13 it is more appropriate to solve the equation

$$
\tilde{\xi}_{2}=\sigma_{0}+3 \alpha V_{1} J_{\tau-\frac{1}{3}}\left(\xi_{1}, \xi_{2}\right)
$$

where we introduced the following notations $J_{\tau-q}\left(\xi_{1}, \xi_{2}\right) \equiv I_{\tau-q}\left(\xi_{1}, \tilde{\xi}_{2}\right)$.

Let us now show that the equation $\xi_{1}(T, \mu)=0$ corresponds to a PT. Assuming that its solution $\mu=\mu_{c}(T)$ exists we study the necessary conditions for a PT occurrence. For this purpose it is sufficient to study the properties of the partial $\mu$-derivatives of the variables $\xi_{1}$ and $\tilde{\xi}_{2}$. The analysis of the partial $T$-derivatives leads to the same results although the obtained equations are more involved. From Eqs. (12) and (17) one finds

$$
\begin{aligned}
& \frac{\partial \xi_{1}}{\partial \mu}=-\frac{\left(1+3 V_{1} \alpha J_{\tau-\varkappa-\frac{1}{3}}\right) \frac{\partial \xi_{L}}{\partial \mu}}{\left(1+V_{1} I_{\tau-1}\right)\left(1+3 V_{1} \alpha J_{\tau-\varkappa-\frac{1}{3}}\right)-3 V_{1}^{2} \alpha J_{\tau-\frac{4}{3}} I_{\tau-\varkappa}}, \\
& \frac{\partial \tilde{\xi}_{2}}{\partial \mu}=-\frac{3 V_{1} \alpha J_{\tau-\frac{4}{3}} \frac{\partial \xi_{L}}{\partial \mu}}{\left(1+V_{1} I_{\tau-1}\right)\left(1+3 V_{1} \alpha J_{\tau-\varkappa-\frac{1}{3}}\right)-3 V_{1}^{2} \alpha J_{\tau-\frac{4}{3}} I_{\tau-\varkappa}} .
\end{aligned}
$$

According to the standard definition of statistical mechanics, if the 1-st derivatives of the pressure of two phases differ from each other at their coexistence curve, then this is a 1-st order PT. If, however, at the two-phase coexistence curve the 1-st derivatives of the pressure of two phases coincide then a PT is of the 2-nd or higher order. Eq. (18) provides one with the difference of the particle number density of gaseous $\rho_{g}$ and liquid $\rho_{L}$ phases, since $\frac{\partial \xi_{L}}{\partial \mu} \equiv \rho_{L} V_{1} / T$ and $\frac{\partial \xi_{1}}{\partial \mu} \equiv\left(\rho_{g}-\rho_{L}\right) V_{1} / T$.

To simplify the analysis we assume that in Eq. (18) $\alpha>1$ and will comment in appropriate places what occurs for the case $\alpha \leq 1$. Then all the sums $I_{\tau-q}\left(0, \xi_{2}\right)$ and $J_{\tau-q}\left(0, \xi_{2}\right)$ are finite for $\xi_{2}>0$. The validity of this statement for the sums $I_{\tau-q}\left(0, \xi_{2}\right)$ follows from a direct inspection of Eq. (14), while for the sums $J_{\tau-q}\left(0, \xi_{2}\right)$ it follows from the fact that for $\alpha>1$ the induced surface tension term $\sigma_{1}$ defined by $(11)$ is always positive and, hence, $\tilde{\xi}_{2}>\xi_{2}$. Therefore, for $\xi_{1}=0$ and $\xi_{2}>0$ the r.h.s. of Eq. 18 does not vanish, i.e. $\frac{\partial \xi_{1}}{\partial \mu} \neq 0$, and, hence, in this case we are dealing with the 1-st order PT and the present model is similar to the simplified SMM [14, 20].

A 1-st order PT occurs also in the case of intersecting curves $\xi_{1}=0$ and $\xi_{2}=0$ at $T=T_{c e p}$ for $\tau>2$. This can be seen form Eqs. (18) and (19), since for $\tau>2$ all sums $I_{\tau-1}(0,0)$ and $I_{\tau-\varkappa}(0,0)$ on the r.h.s. of these equations are finite. Therefore, at $T=T_{\text {cep }}$ this case exhibits not a critical point, but a critical line due to different particle densities of the gaseous and liquid phases. Such phase diagrams are known from the literature [5, 28, 33], although there are no such empirical examples.

A different situation arises in the case $\tau \leq 2$, if on the curve $\xi_{1}=0$ one also has a point where $\xi_{2}=0$. Then the sums $J_{\tau-q}\left(0, \xi_{2}\right)$ are finite since $\tilde{\xi}_{2}>\xi_{2}$, but the sums $I_{\tau-1}(0,0)$ and $I_{\tau-\varkappa}(0,0)$ 
diverge. Thus, for $\tau \leq 2$ the sum $I_{\tau-1}(0,0) \rightarrow \infty$ and in this case $\frac{\partial \xi_{1}}{\partial \mu}=0$ and, hence, the model exhibits a PT of the 2 -nd or higher order. Note that by the adopted assumptions $\varkappa<1$, the sum $I_{\tau-1}(0,0)$ is the most divergent one in Eq. (18). After evaluating the second derivative of $\xi_{1}$ and taking the limit $\xi_{2} \rightarrow+0$, one obtains

$$
\frac{\partial^{2} \xi_{1}}{\partial \mu^{2}} \rightarrow-\frac{\partial \xi_{L}}{\partial \mu} \cdot \frac{1}{V_{1} I_{\tau-1}^{2}}\left[\frac{\partial \xi_{1}}{\partial \mu} I_{\tau-2}+\frac{1}{\alpha} \frac{\partial \tilde{\xi}_{2}}{\partial \mu} I_{\tau-1-\varkappa}\right] \rightarrow-\left[\frac{\partial \xi_{L}}{\partial \mu}\right]^{2} \cdot \frac{I_{\tau-2}(0,0)}{V_{1}^{2}\left[I_{\tau-1}(0,0)\right]^{3}}
$$

where in the last step of evaluation we took into account that the sum $I_{\tau-2}(0,0)$ is more divergent than the sum $I_{\tau-1-\varkappa}$ and that according to Eq. (19)

$$
\frac{\partial \tilde{\xi}_{2}}{\partial \mu} \rightarrow \frac{\partial \xi_{1}}{\partial \mu} \cdot \frac{3 V_{1} \alpha J_{\tau-\frac{4}{3}}}{1+3 V_{1} \alpha J_{\tau-\varkappa-\frac{1}{3}}}
$$

To complete the evaluation of Eq. 20 we need to analyze the behavior of the sums $I_{\tau-q}\left(0, \xi_{2}\right)$ in the limit $\xi_{2} \rightarrow 0$. This can be done either rigorously using the integral representations of these sums developed in [20] or employing a more straightforward way used below. Consider first the large, but finite number of nuclear fragment sorts $N$ in all sums (14). Then each sum is finite for any finite value of parameters and, hence, one can find the limit $\xi_{1}=0$ and $\xi_{2}=0$ without any trouble and calculate the sum as an integral

$$
\begin{aligned}
I_{\tau-q}(0,0) & =\lim _{N \rightarrow \infty}\left[\frac{m T}{2 \pi}\right]^{\frac{3}{2}}\left[b_{1}(T)+\sum_{k=2}^{N} \frac{1}{k^{\tau-q}}\right]=\left[\frac{m T}{2 \pi}\right]^{\frac{3}{2}} b_{1}(T)+\lim _{N \rightarrow \infty}\left[\frac{m T}{2 \pi}\right]^{\frac{3}{2}} \int_{2}^{N} d x x^{q-\tau} \\
& =\left[\frac{m T}{2 \pi}\right]^{\frac{3}{2}} b_{1}(T)+\left[\frac{m T}{2 \pi}\right]^{\frac{3}{2}} \cdot \lim _{N \rightarrow \infty}\left\{\begin{array}{l}
\frac{1}{\tau-q-1} 2^{q+1-\tau}, \quad \text { for } \quad q<\tau-1, \\
\ln \left[\frac{N}{2}\right], \quad \text { for } q=\tau-1, \\
\frac{1}{q+1-\tau} N^{q+1-\tau}, \text { for } q>\tau-1 .
\end{array}\right.
\end{aligned}
$$

Applying these results to the case $\tau \leq 2$, one finds that in the limit $N \rightarrow \infty$ both sums $I_{\tau-2}(0,0) \sim$ $N^{3-\tau}$ and $I_{\tau-1}(0,0) \sim N^{2-\tau}$ diverge, but the second derivative $\frac{\partial^{2} \xi_{1}}{\partial \mu^{2}} \sim N^{2 \tau-3}$ in 20 vanishes only for $\tau<\frac{3}{2}$. Therefore, in this model the 2-nd order PT exists for $\xi_{1}=0, \xi_{2}=0$ and $\frac{3}{2} \leq \tau \leq 2$, whereas for $\tau<\frac{3}{2}$ there is a PT of the 3-rd or higher order. Similarly, studying the higher order $\mu$-derivatives of functions $\xi_{1}$ for intersecting (or matching) curves $\xi_{1}=0$ and $\xi_{2}=0$, one can get the following expression

$$
\frac{\partial^{n} \xi_{1}}{\partial \mu^{n}} \sim-\left[\frac{\partial \xi_{L}}{\partial \mu}\right]^{n} \cdot \frac{\left[I_{\tau-2}(0,0)\right]^{n-1}}{V_{1}^{n}\left[I_{\tau-1}(0,0)\right]^{2 n-1}},
$$

for the $n$-th derivative $(n=3,4,5, \ldots)$. In this case there exist the $n$-th order PT for $\frac{n+1}{n} \leq \tau<\frac{n}{n-1}$. The last result can be applied for $n=2$ as well, although $\tau=2$ is a special case of the 2-nd order PT that is not described by this formula. Thus, the minimal value of the Fisher index is $\tau=1$ and in this case one may expect a Kosterlitz-Thouless PT of infinite order. Note that this situation is different from the simplified SMM analytical solution [14, 20] for which the critical endpoint exists for any $\tau<1$, but it is similar to the gas of bags with surface tension model which has a tricritical endpoint [28].

Now it is necessary to mention that for $\alpha=1$ the whole previous consideration should be modified, since in this case for $\xi_{1}=0$ and $\xi_{2}=\tilde{\xi}_{2} \rightarrow+0$ the leading term in Eqs. (18) and (19) is not $I_{\tau-1}\left(0, \xi_{2}\right)$, but the product $J_{\tau-\frac{4}{3}}\left(0, \xi_{2}\right) I_{\tau-\varkappa}\left(0, \xi_{2}\right)$. Our numerical analysis showed that although in this case there exists a tricritical endpoint for $\xi_{1}=0$ and $\xi_{2}=0$ in its vicinity the denominator of expressions (18) and (19) always changes a sign leading to an unphysical situation, when the 
negative values of particle density of a gaseous phase coexist with a liquid phase of positive particle density. For $\alpha<1$ the model does not have the (tri)critical endpoint, since from $\xi_{1}=0$ and $\xi_{2}=0$ it follows that $\tilde{\xi}_{2}<0$ due to positive values of $\sigma_{1}$ in $(16)$. However, for $\tilde{\xi}_{2}<0$ and $\xi_{1}=0$ the r.h.s. of (17) diverges to $+\infty$, whereas its left hand side is negative. Consequently, such solutions do not exist. Therefore, in this work we consider only the case $\alpha>1$ which has no such defects. In fact, the values $\alpha>1$ show that the third and higher order virial coefficients are less important for the induces surface tension $\sigma_{1}$ at the tricritical endpoint vicinity and, hence, compared to the second virial coefficients their contribution to $\sigma_{1}$ is suppressed due to the inequality $\alpha>1$.

It is easy to show that for any finite value of the function $\xi_{L}$ there are no solutions with $\xi_{1}<0$. Let us assume that such a solution of the system (12)-(14) exists. Then for any value of $\tau$ and $\xi_{2}$ the sum $I_{\tau}\left(\xi_{1}, \xi_{2}\right)$ diverges to $+\infty$ and, hence, from (12) one gets that $\xi_{1} \rightarrow+\infty$, i.e. we arrive at a contradiction. However, the present model contains the solutions with negative surface tension coefficients, i.e. for $\xi_{2}<0$. It is clear that for $\xi_{2}<0$ there is no PT, since in this case the solution of the system (12)-(14) exists only for $\xi_{1}>0$. Compared to the simplified SMM this is entirely new case, which at first glance may look unphysical. However, as it is argued in [28, 35] there is nothing wrong or unphysical with the negative values of surface tension coefficient, since in the grand canonical ensemble the quantity $\xi_{2} k^{\varkappa}$ is the surface free energy $f_{\text {surf }}=e_{\text {surf }}-T s_{\text {surf }}$ of the nuclear fragment of mean volume $k V_{1}$, were $e_{\text {surf }}$ and $s_{\text {surf }}$ are the surface energy and entropy. Therefore, $\xi_{2}<0$ means that the surface entropy contribution simply exceeds the surface energy part, i.e. $T s_{\text {surf }}>e_{\text {surf }}$ and then $f_{\text {surf }}<0$. It can be shown on the basis of exactly solvable model of surface deformations [36, 37] that negative values of the surface free energy appear as a consequence of very large number of non-spherical configurations at high temperatures.

We would like to point out that negative values of the surface tension coefficient may provide us a physical reason preventing the condensation of small droplets into a liquid phase (an infinite droplet) at supercritical temperatures, and naturally explain the existence of a cross-over transition in ordinary liquids [34] as well as in QGP [28, 35, 38, 40]. This may lead to an appearance of surfaces with the fractal dimension.

\section{PHASE DIAGRAM OF THE NUCLEAR LIQUID-GAS PT}

In the original SMM the pressure of nuclear liquid is expressed as $p_{L}=\frac{\mu+W(T)}{V_{1}}$. Such a parameterization corresponds to an incompressible liquid, since the isothermal compressibility $\left.K_{T} \equiv \frac{1}{\rho} \frac{d \rho}{d p}\right|_{T}$ is zero in this case. Obviously, this fact is in contradiction with the existing experimental information on the compressibility modulus. Here we propose another liquid pressure parametrization which provides a more realistic description. Since the necessary condition for a non-zero compressibility is $\frac{\partial^{2} p_{L}}{\partial \mu^{2}} \neq 0$, we assume that liquid phase pressure has a form [34]

$$
p_{L}=\frac{W_{F m}(T)+\mu+W_{0}+a_{\nu}\left[\mu+W_{0}\right]^{\nu}}{V_{1}}, \quad \text { for } \quad \nu=2,3,4,
$$

where $a_{\nu}$ is a constant which should be found from a normalization condition (more details can be found in Appendix A). Of course, one could use more complicated parameterizations of the liquid phase pressure, but below we show that even such a simple modification leads to a rather rich structure of the nuclear phase diagram.

Using the definition of particle density for the liquid phase, $\rho_{L}=\frac{\partial p_{L}}{\partial \mu}$, one gets

$$
\rho_{L}=\frac{1+a_{\nu} \nu \tilde{\mu}^{\nu-1}}{V_{1}},
$$

where an effective chemical potential is denoted as $\tilde{\mu} \equiv \mu+W_{0}$. One can identically rewrite Eq. 
TABLE I: The model parameters providing the condition $\rho_{c e p}=\rho_{0} / 3$.

\begin{tabular}{|c|c|c|c|}
\hline model parameter & \multicolumn{3}{|c|}{ value } \\
\hline$\tau$ & 1.9 & 1.9 & 1.9 \\
\hline$\nu$ & 2 & 3 & 4 \\
\hline$a_{\nu}\left(\mathrm{MeV}^{1-\nu}\right)$ & $1.261 \cdot 10^{-2}$ & $-4.414 \cdot 10^{-4}$ & $1.803 \cdot 10^{-5}$ \\
\hline
\end{tabular}

(24) in a more familiar way

$$
p_{L}=\rho_{0}\left[W_{F m}(T)+\left(\frac{\rho_{L}-\rho_{0}}{\nu a_{\nu} \rho_{0}}\right)^{\frac{1}{\nu-1}}\left[1+\frac{\rho_{L}-\rho_{0}}{\nu \rho_{0}}\right]\right],
$$

which for $\nu=2$ is a second order polynomial in $\rho_{L}$, while for other integer values of the parameter $\nu$ Eq. (26) looks more sophisticated.

An important practical purpose to employ the liquid gas pressure (24), (26) is to reduce the standard SMM critical density $\rho_{\text {cep }}$ from the normal nuclear density $\rho_{0}=1 / V_{1} \simeq 0.16 \mathrm{fm}^{3}$ to the value $\rho_{c e p}=\rho_{0} / 3$ which is typical for the liquid-gas PTs [24]. The condition $\rho_{c e p}=\rho_{0} / 3$ is an additional constraint on the developed EoS. Using (25) and requiring that at the critical endpoint with the coordinates $\left(T_{c e p} ; \mu_{c e p}\right)$ the condition $\rho_{L}\left(\mu_{c e p}\right)=\rho_{c e p}=\rho_{0} / 3$, one finds the normalization constant $a_{\nu}$

$$
a_{\nu}=\left[\frac{\rho_{c e p}}{\rho_{0}}-1\right] \frac{\tilde{\mu}_{c e p}^{1-\nu}}{\nu}=-\frac{2 \tilde{\mu}_{c e p}^{1-\nu}}{3 \nu} .
$$

Since at the critical endpoint $\xi_{1}=\xi_{2}=0$, Eq. (12) can be written as

$$
a_{\nu} \tilde{\mu}^{\nu}+\tilde{\mu}+W\left(T_{c e p}\right)-T_{c e p} V_{1} I_{\tau}(0,0)=0 .
$$

Thus, the chemical potential at critical endpoint $\mu_{\text {cep }}$ can be found from Eq. (28) directly. Indeed, substituting the standard value of the critical temperature $T_{c e p}=18 \mathrm{MeV}$ into (28) for an unknown $\tilde{\mu}=\tilde{\mu}_{c e p}$ and accounting for the normalization condition (27), one finds

$$
\tilde{\mu}_{c e p}\left[1+\frac{1}{\nu}\left(\frac{\rho_{c e p}}{\rho_{0}}-1\right)\right]=T_{c e p} V_{1} I_{\tau}(0,0)-W\left(T_{c e p}\right) .
$$

Note that Eq. (29) has a single solution for $\tilde{\mu}=\tilde{\mu}_{c e p}$ ! Finding the $\mu_{c e p}$ values for different $\nu$, one can determine the normalization constant $a_{\nu}$ from Eq. (27). Their values are given in Table 1. However, if one substitutes now the obtained value of $a_{\nu}$ into Eq. (28) and solves it for $\tilde{\mu}$, then one obtains several solutions for $\mu_{c e p}$ ! For instance, for $\nu=2$ one can easily check that there are two solutions $\tilde{\mu}_{c e p 1} \simeq-26.5 \mathrm{MeV}$ and $\tilde{\mu}_{c e p 2} \simeq-54 \mathrm{MeV}$. Note that an additional solution $\tilde{\mu}_{c e p 2}$ is unphysical since it corresponds to the negative particle density of the liquid.

A careful analysis of Eqs. (12) and (17) shows that for any integer $\nu>1$ at $T_{\text {cep }}$ there is a curve of the 2-nd order PT that always begins at the line of the 1-st order PT (see Figs. 1, 4 ). Therefore, all the critical endpoints of this model are, in fact, the tricritical points. This feature of the present model is similar to the simplified SMM solution for $1<\tau \leq 2$ [14, 20], but its location is entirely different. In the simplified SMM the 2-nd order PT always exists at the particle number density $\rho=\rho_{0}$ and for temperatures $T \geq T_{c e p}=18 \mathrm{MeV}$, whereas in the present model such a PT occurs at the isotherm $T=T_{c e p}=18 \mathrm{MeV}$ (see Figs. 1 and 2) along which the particle number density changes with the pressure, as one can see from Figs. 3 and 4 . As one can see from Fig. 2 a variation of the parameter $\tau$ from 1.8 to 2.2 leads to a negligible change of the 1-st order PT curve in the $T-\mu$ plane and to a small variation of the nil curve of surface tension coefficient. As one can seen from Fig. 2, in the limiting case $\tau \rightarrow \infty$ the nil curve of surface tension coefficient becomes an isotherm $T=T_{c e p}$. 
Note that the shape of phase diagrams of the present model in the $\rho-p$ plane shown in Figs. 3 and 4 look very similar to that ones for real liquids [24], although the critical isotherms at the vicinity of the tricritical endpoint shown in Figs. 3 and 4 are rather flat compared to the mean-field calculations of Ref. [39]. This is due to the different values of the critical index $\delta$ which in the mean-field models is $\delta_{m f}=3$ [24] whereas in the present model this index can be roughly estimated as $\delta \simeq 6$ for $\tau=1.9$ and $\delta \simeq 5$ for $\tau=1.8$. A more accurate estimate for the index $\delta$ can be provided by the analytical calculations of the critical exponents, which is out of the scope of the present paper.

To constrain the choice of model parameters below we apply the L. van Hove axioms of statistical mechanics [32], which shortly can be formulated as follows. Suppose that we are able to evaluate the exact $N$ particle partition function $Q_{N}(V, T)$ of the given system.

1. Then in the thermodynamic limit, i.e. when $N \rightarrow \infty$ and $V \rightarrow \infty$ while the density $\rho=N / V$ stays constant, the thermodynamic pressure defined as

$$
p(\rho, T)=T\left(\frac{\partial \ln Q_{N}}{\partial V}\right)_{N, T},
$$

is a strictly non-negative quantity.

2. The slope $\left(\frac{\partial p}{\partial \rho}\right)_{T}$ of any isotherm in $(\rho, p)$ plane defined by 30 is never negative. The limiting case corresponds to an existence of "flat" region (regions) in which $\left(\frac{\partial p}{\partial \rho}\right)_{T}=0$ and, hence, the system becomes infinitely compressible. The existence of such regions in the $(\rho, p)$ plane corresponds to the coexistence of two or more phases in the given system.

3. The presence of the absolutely flat part of an isotherm with $\left(\frac{\partial p}{\partial \rho}\right)_{T} \equiv 0$ with the mathematical singularities at its ends, is a consequence of the limit $N \rightarrow \infty$. If $N$ were finite, but large, then the pressure (30) would be free from mathematical singularities. The usual sharp corners of an isotherm would be round off and, at the same time, the usual flat part of the isotherm would not be really flat, but instead it would have a small positive slope $\left(\frac{\partial p}{\partial \rho}\right)_{T}$.

These axioms play an important role for the approximate partition functions too. For example, just the second axiom above requires to use the Maxwell construction [24, 32] in order to get rid of the isotherms of the Van der Waals type for which $\left(\frac{\partial p}{\partial \rho}\right)_{T}<0$ in the phase transition region. As we demonstrate below the present model obeys these axioms for some choice of the model parameters.

For a numerical evaluation of Eqs. (12) and (17) we used the following parameterization of the eigen surface free energy coefficient $\sigma_{0}(T)$

$$
\sigma_{0}(T)=\sigma_{01}-\sigma_{02}\left(\frac{T}{T_{c e p}}\right)^{\zeta},
$$

where the eigen surface tension at $T=0$ is taken from the standard SMM parameterization $\sigma_{01}=18$ $\mathrm{MeV}$ and $\sigma_{02}>0$ and $\zeta$ are some constants. We found that for the parameter $\zeta \geq 1$ in the vicinity of the tricritical point the total surface tension coefficient always vanishes as the first power of the difference $T-T_{c e p}$, i.e. the temperature dependence of the surface tension of the present model is similar to the FDM [18] and to the exactly solvable model of surface deformations [36, 37]. Therefore, the value $\zeta=1$ was used in actual calculations, while the constant $\sigma_{02}$ was determined from Eq. (17) to provide the critical endpoint existence at $\xi_{2}=0$. For the parameter $\alpha=1.5$ used in our calculations we find $\sigma_{02} \simeq 24.76 \mathrm{MeV}$ for any $\nu>1$. Thus, at the critical endpoint Eq. (17) can be explicitly written as

$$
3 V_{1} I_{\tau-\frac{1}{3}}\left(0,(1-\alpha) \sigma_{0}\left(T_{c e p}\right)\right)=-\sigma_{0}\left(T_{c e p}\right) .
$$


On the one hand, this equation can be considered as a condition to determine $\sigma_{02}$ for a given value of $T_{c e p}$, but, on the other hand, for a fixed value of the constant $\sigma_{02}$ it can be regarded as an equation for unknown value of $T_{\text {cep }}$. Our numerical analysis shows that for $\nu=2$ and $\nu=4$ there exists a single solution for $T_{\text {cep }}$.

For $\nu=3 \mathrm{Eq}$. (28) has three branches of solutions at $T_{\text {cep }}=18 \mathrm{MeV}$ which correspond to three tricritical points at this temperature, but only one of them is a physical one. The solution with the lowest value of chemical potential $\mu_{c e p}$ which corresponds to the negative particle density is similar to the corresponding PT curves for $\nu=2$. The two remaining solution have, respectively, either negative values of the chemical potential $\mu_{c}(T) \leq-W_{0}$ along the coexistence or positive values of $\mu_{c}(T)>0$ for $T \leq T_{c e p}$. In order to understand which solution is the physical one, we apply the second L. van Hove axiom given above. The latter requires that the inverse compressibility modulus $1 / K_{T}$ of liquid phase alone should not be negative. From this requirement one gets

$$
K_{T} \equiv \frac{\nu(\nu-1)}{V_{1} \rho_{L}^{2} \tilde{\mu}^{2}} \cdot a_{\nu} \tilde{\mu}^{\nu} \geq 0 \quad \Longrightarrow \quad a_{\nu} \tilde{\mu}^{\nu} \geq 0
$$

Since for $\nu=3$ the condition $\rho_{c e p} \simeq \rho_{0} / 3$ can be obeyed only for negative value of the parameter $a_{3}$, i.e. $a_{3}<0$, then according to (33) the positive compressibility of this liquid corresponds to the inequality $\tilde{\mu} \leq 0$, i.e. $\mu \leq-W_{0}$. Therefore, any additional tricritical point that corresponds to positive value of chemical potential $\mu$ is unphysical. This indicates that for $\nu=3$ the liquid phase pressure (24) is not very realistic, since it is not able to describe the observed experimental states at high particle number densities. Actually, the same arguments are applicable to the odd powers $\nu \geq 3$ and, hence, the corresponding EoS are also unphysical.

This is also the case for the even values of power $\nu$ in (24), except for the case $\nu=2$. This follows from the analysis of the nuclear incompressibility modulus, $K_{0} \equiv 9\left(\frac{\partial p_{L}}{\partial \rho_{L}}\right)_{T}$, at normal nuclear density and vanishing temperature. Indeed, from (24) one can find explicitly

$$
K_{0}=\frac{9}{\nu(\nu-1) a_{\nu} \tilde{\mu}^{\nu-2}} .
$$

This expression shows that for the adopted parameterization of liquid phase EoS the coefficient $K_{0}$ is finite only for $\nu=2$ and $K_{0}(\nu=2)=\frac{9}{2 a_{2}} \simeq 357 \mathrm{MeV}$, while for any power $\nu>2$ the incompressibility modulus (34) diverges, because $\tilde{\mu}=0$ at $T=0$ (for more details see Appendix A). Experimental values of the nuclear incompressibility modulus are quoted as $K_{0}^{\text {exp }} \simeq 230 \pm 30 \mathrm{MeV}$ [42 44], but theoretical models with larger value up to $350-380 \mathrm{MeV}$ are also known. For instance, the Skyrme force model SIII, which is able to well describe the empirical properties of many nuclei [45], has the value of the incompressibility modulus $K_{0} \simeq 355 \mathrm{MeV}$.

The obtained value of $K_{0}(\nu=2)$ can, of course, be reduced to $K_{0}(\nu=2) \simeq 250 \mathrm{MeV}$ by choosing $\mu_{0} \simeq-6 \mathrm{MeV}$ in the parameterization (45), but this immediately leads to a strong decrease of the particle number density of the liquid at the phase equilibrium curve at $T=0$ to $\rho_{L} \simeq 0.7 \rho_{0}$ which is much more unrealistic than the large value of the nuclear incompressibility modulus.

From the discussion above it is clear that only the liquid phase pressure (24) with $\nu=2$ fulfills the second L. van Hove axiom. A close inspection shows that the incompressibility modulus of the gaseous phase is always positive (see also [14]). Since the present model is an exactly solvable, it automatically leads to the Gibbs criterion of phase equilibrium [5, 14, 15, 20, 28]. Moreover, in the mixed phase the pressure derivative with respect to $\rho$ at constant $T$ is exactly zero, i.e. $\left(\frac{\partial p}{\partial \rho}\right)_{T}=0$, i.e. the proposed model EoS has no regions of negative $\left(\frac{\partial p}{\partial \rho}\right)_{T}$ values (see isotherms in Figs. 3 and 4 ). Therefore, the present model EoS obeys the second axiom of statistical mechanics [32] everywhere. Although the liquid phase pressure (24) can be negative, but the gaseous phase pressure is always positive and, hence, in this region the phase equilibrium cannot be achieved and in this case the gaseous phase dominates. Therefore, this EoS obeys the first axiom of statistical mechanics [32] 
too. We would like to stress that the present model has the same physical mechanism of the PT generation as the simplified SMM [5, 14, 15] and, hence, it can be solved analytically for finite volumes using the methods suggested in [41]. For finite volumes the number of sorts of fragments $N$ in Eqs. (10) and (11) is restricted from above $N \leq V / V_{1}$ and because of that the essential singularity of the isobaric partition disappears [41] and a PT is washed out [5, 41. Moreover, for finite, but large number of particles $N$ inside the finite volume analog of mixed phase the derivative

$\left(\frac{\partial p}{\partial \rho}\right)_{T}$ of thermodynamic pressure 30 vanishes as $c^{2} / N$. Therefore, the present model EoS obeys the third L. van Hove axiom 32 as well.

\section{MANIFESTATION OF NEGATIVE SURFACE TENSION VALUES}

In this section we study the fragment size distributions in different parts of the phase diagram in order to elucidate the role of the negative surface tension coefficient. But first we consider the gas of nuclear fragments with nonnegative surface tension coefficient, i.e. for the dimensionless surface term $\xi_{2} \geq 0$. In this case the fragment size distribution is proportional to

$$
\omega_{\text {gas }}(k)=\exp \left[-\xi_{1} k-\left|\xi_{2}\right| k^{\varkappa}-\tau \ln k\right],
$$

where $k$ is the number of nucleons in a fragment. It is monotonically decreasing function of the fragment size $k$, since $\xi_{1}>0$ is valid everywhere except for the PT curve, where $\xi_{1}=0$. As one can see from Fig. 5, for all temperatures below the critical one the low $k$ behavior is governed by the Fisher exponent $\tau$ and by the surface term which is proportional to $\xi_{2}$, whereas at large values of $k$ the fragment size attenuation is solely determined by the bulk term which is proportional to $\xi_{1}$. These fragment size distributions are qualitatively similar to that ones found recently in [46] for the nuclear matter EoS neutralized by the electrons in the stellar environments. A true power law of the fragment size distribution corresponds to the tricritical endpoint, for $\xi_{1}=0$ and $\xi_{2}=0$. In Fig. 5 one can also see the effective power law of the fragment size distributions for temperatures which are very close to $T_{c e p}$. A similar behavior can be seen at lower temperatures, if $\xi_{1} \ll 1$ and $\xi_{2} \ll 1$.

In the mixed phase the bulk term vanishes due to the PT condition $\xi_{1}=0$ and, hence, the fragment distribution of the gas phase acquires the form

$$
\omega_{\text {gas }}^{M}(k)=\exp \left[-\left|\xi_{2}\right| k^{\varkappa}-\tau \ln k\right],
$$

which is governed by the Fisher term (at small $k$ only) and by the surface term (see Fig. 6). In addition to the gaseous phase distribution (36) the mixed phase contains an infinite fragment representing the liquid phase. Its distribution function corresponds to the Kronecker $\delta$-function. In order to make a comparison with the results reported in [46] we have normalized the fragment size distributions in the mixed phase to the total number of 500 nucleons. In Fig. 6 we present results for the fixed particle number density $\rho=\frac{\rho_{0}}{3}$. As one can see, at low temperatures the gas of fragments is practically absent and, hence, almost whole matter belongs to the liquid fragment with the size of almost 500 nucleons. For higher temperatures the size of liquid fragment decreases and the gaseous phase appears. At the tricritical point about $65 \%$ of the matter belongs to the liquid fragment, while the rest belongs to the gas, which demonstrates a clear power law. Such a behavior of the fragment size distributions in the mixed phase is qualitatively similar to the one found in [46] inside the mixed phase, see Figs. 4 and 6 in [46]. The main difference with the results of [46] is that the present model employes the grand canonical ensemble in thermodynamic limit, whereas [46] is dealing with the canonical ensemble for a finite system. In the latter case the size of largest fragment, which represents the liquid phase, fluctuates from event to event and, hence, instead of the Kronecker $\delta$-function distribution one gets a Gaussian one with a finite width.

A principally new type of the fragment size attenuation corresponds to the region with negative values of the total surface tension coefficient, i.e. for $\xi_{2}<0$. In this case the unnormalized 
distribution of nuclear fragments has the form

$$
\omega(k)=\exp \left[-\xi_{1} k+\left|\xi_{2}\right| k^{\varkappa}-\tau \ln k\right]
$$

i.e. it has the local minimum at some value $k_{\min }$ and the local maximum at $k_{\max }>k_{\min }$. This can be shown by inspecting the logarithmic derivative of $\omega(k)$ with respect to $k$. The extremum condition for such a derivative is given by the equation

$$
\left.\frac{\partial \ln \omega(k)}{\partial k}\right|_{k=k_{E}}=-\xi_{1}+\varkappa \frac{\left|\xi_{2}\right|}{k_{E}^{1-\varkappa}}-\frac{\tau}{k_{E}}=0 \Rightarrow k_{E}=\left[\frac{\varkappa\left|\xi_{2}\right|}{\xi_{1}+\frac{\tau}{k_{E}}}\right]^{\frac{1}{1-\varkappa}},
$$

where the extremum is reached for $k=k_{E}$. Let us show now that the expression for $k_{E}$ in $(38)$ has two positive solutions. In first case we assume that $\xi_{1} \ll \frac{\tau}{k_{E}}$, which may occur for small values of $k_{E}$. Then neglecting the term $\xi_{1}$ in the above expression for $k_{E}$ one finds

$$
k_{\text {min }} \simeq\left[\frac{\tau}{\varkappa\left|\xi_{2}\right|}\right]^{\frac{1}{\varkappa}}
$$

The analysis of the second derivative of $\ln \omega(k)$ with respect to $k$

$$
\left.\frac{\partial^{2} \ln \omega(k)}{\partial k^{2}}\right|_{k=k_{\min }}=-\varkappa(1-\varkappa) \frac{\left|\xi_{2}\right|}{k_{\min }^{2-\varkappa}}+\frac{\tau}{k_{\min }^{2}}=\frac{\varkappa \tau}{k_{\min }^{2}}>0,
$$

shows that this derivative is always positive, i.e. there is a local minimum, for $\varkappa>0$. Note that Eq. (39) allows one to estimate the dimensionless surface term $\xi_{2} \simeq-\frac{\tau}{\varkappa k_{m i n}^{\varkappa}}$, if the position of the local minim is known. the form

In the opposite case, if $\xi_{1} \gg \frac{\tau}{k_{E}}$, which occurs for large values of $k_{E}$, the solution for $k_{E}$ takes

$$
k_{\max } \simeq\left[\frac{\varkappa\left|\xi_{2}\right|}{\xi_{1}}\right]^{\frac{1}{1-\varkappa}} .
$$

The second derivative of $\ln \omega(k)$ with respect to $k$ can be written as

$$
\left.\frac{\partial^{2} \ln \omega(k)}{\partial k^{2}}\right|_{k=k_{\max }}=-\varkappa(1-\varkappa) \frac{\left|\xi_{2}\right|}{k_{\max }^{2-\varkappa}}+\frac{\tau}{k_{\max }^{2}}=-\frac{1}{k_{\max }}\left[\xi_{1}(1-\varkappa)-\frac{\tau}{k_{\max }}\right] .
$$

This derivative is negative for $\xi_{1}(1-\varkappa)>\frac{\tau}{k_{\max }}$. This inequality cannot be fulfilled only for $(1-\varkappa) \ll$ 1 , whereas for the typical SMM value $\varkappa \simeq \frac{2}{3}$ it is fulfilled due to adopted assumption $\xi_{1} \gg \frac{\tau}{k_{\max }}$. Thus, at $k \simeq k_{\max }$ the fragment distribution (37) has a local maximum. The size distributions with the saddle-like shape which have both a local minimum and a local maximum are clearly seen in Figs. (7) and (8). Our analytical estimates are well supported by the numerics.

Combining the expressions (39) and (41), one can get an approximate relation between the mass numbers of fragments that correspond to these two extrema

$$
k_{\min }^{\varkappa} k_{\max }^{1-\varkappa} \simeq \frac{\tau}{\xi_{1}}
$$

which allows one to estimate the dimensionless bulk term $\xi_{1}$ for a given fragment mass distribution which has two extrema.

Another distinctive feature of the fragment size distributions with the negative surface tension coefficient is a presence of a quasi-power law for a wide range of fragment sizes below $k_{\text {min }}$. It appears due to the fact that $k_{\min }$ and $k_{\max }$ are large because of small values of bulk $\xi_{1}$ and surface $\xi_{2}$ terms. If the fragment size exceeds $k_{\max }$, then the bulk term in 37 dominates and the distribution $\omega(k)$ becomes exponential, which in a double logarithmic scale looks like a fast fall off. 
Note that a quasi-power law persists to exist even, if both a minim and a maximum move to each other and become very shallow. The condition of their disappearance is just $k_{\min }=k_{\max }$, which with the help of (43) can be represented as

$$
\frac{\tau}{\xi_{1}} \simeq\left[\frac{\tau}{\varkappa\left|\xi_{2}\right|}\right]^{\frac{1}{\varkappa}}
$$

In this case a quasi-power law at small values of $k$ changes to a slightly convex shape which at larger values of $k$ acquires a strong concave shape and then it turns into an exponential all off. From (44) it is easy to find that the reason for such a behavior is just a cancellation of the bulk and surface contributions in the vicinity of $k=k_{\min }=k_{\max }$.

It is necessary to mention that the saddle-like mass distributions were reported in Ref. [47] where the authors numerically studied the multifragmentation scenario for the ensemble of classical particles interacting via the Lennard-Jones potential. Thus, the full curve in Fig. 2 of [47] is very similar to the distributions with the negative surface tension coefficient discussed above, although its extrema are not so well pronounced as the ones shown in Figs. 7 and 8 . It is, of corse, difficult to directly compare our results for the fragment size distributions with the ones found in [47] and to make some definite conclusions, since the authors of [47] either studied small systems (189 particles) in which the finite size effects are strong (see Fig. 2 in [47]) or they have low statistics for the power law shown in Fig. 8 of [47]. Nevertheless, the authors of [47] claimed to find a curve of power law size attenuations with nearly constant energy per particle. Note that such a behavior is typical for the present model along the curve of the 2-nd order PT. In [47] it was also found that in the course of expansion from the initially dense state to a dilute state the fragments are highly nonspherical and they have fractal surfaces. The above analysis of the gaseous fragment size distributions shows us that the fractals which appear at the tricritical point or at the states of the 2-nd order PT correspond to a power law dependence on the size of fragments, whereas the fractals associated with the negative values of the surface tension coefficient manifest themselves in the nonmonotonic fragment size distributions of the saddle-like shape. Therefore, it is quite possible that the negative surface tension is responsible for the peculiar and nonmonotonic fragment size distributions reported in [47, but for a more definite conclusion a detailed comparison between the two models is needed.

\section{CONCLUSIONS}

In the present work we propose a new SMM formulation based on the consistent treatment of the second virial coefficients for the ensemble of nuclear fragments. Such a virial expansion allows us to explicitly account for the many-body effects. Our analysis shows that interaction between the nuclear fragments induces an additional contribution into the surface tension free energy. It is shown that by a proper choice of the temperature dependence of the full surface tension the standard SMM which accounts only for the proper volumes of fragments is able to correctly reproduce the low density virial expansion up to the second order. This resolves an old puzzle of why the SMM is so good at low densities, although it employes the hard core repulsion approximation which is suited for high densities only.

The present model, however, leads to an additional equation for the induced surface tension coefficient, which at the moment accounts only for the repulsion between the nuclear fragments. In order to simplify the presentation of our idea and to make easier an analysis of the model phase diagram, here we assumed that the effects of attraction between the nuclear fragments are implicitly accounted in the temperature dependent surface tension coefficient of the fragments. However, the treatment of the model with an attraction between the fragments will be our next step.

Another important result of the present work is extension of the previous model for the case of a compressible nuclear liquid. This is achieved by introducing an additional $\mu$-dependent term in the expression for liquid phase pressure. Note that the obtained model EoS for $\nu=2$ obeys the basic axioms of the statistical mechanics formulated by L. van Hove. It does not lead to an appearance 
of the non-monotonic isotherms in the mixed phase region which are typical for the mean-field models. A direct consequence of the finite liquid compressibility is that the present model allows us to generate the tricritical endpoint at $\rho_{c e p}=\rho_{0} / 3$, which is a typical value of critical density for the liquid-gas PTs in the ordinary liquids. This novel feature makes the present model more realistic than the standard SMM.

The range of the Fisher parameter in this model is $\tau \geq 1$, whereas in the simplified SMM all values of the parameter $\tau$ are allowed. The other important difference with the simplified SMM is that in the present model there exist the tricritical points only and this is possible for $1 \leq \tau \leq 2$. Thus, our analysis showed that in the present model each 1-st order PT curve is ended at the curve of the 2-nd (or higher) order PT. It is found that the 2-nd order PT in the tricritical point exists for $\frac{3}{2} \leq \tau \leq 2$, while the $n$-th $(n=3,4,5, \ldots)$ order PT in a tricritical point exist for $\frac{n+1}{n} \leq \tau<\frac{n}{n-1}$.

For $\tau>2$ we found that, in contrast to the simplified SMM, the 1-st order PT exists at $T \leq T_{\text {cep }}$ and, hence, in this case instead of the critical endpoint the model exhibit a critical line. Therefore, the physical range of the Fisher parameter $\tau$ should be constrained by $\frac{3}{2} \leq \tau \leq 2$.

Our analysis of the fragment size distributions in the region of negative surface tension coefficient showed that these distributions have an unusual saddle-like shape. We established a simple relation between the local minimum position in the fragment size $k_{\text {min }}$ and the dimensionless surface term $\xi_{2} \simeq-\frac{\tau}{\varkappa k_{m i n}^{\varkappa}}$ which can be used to estimate the $\xi_{2}$ value directly from the fragment size distribution. We would like to stress that the distributions of a similar shape were observed in molecular dynamical studies of multifragmentation for the particles interacting via the Lennard-Jones potential [47].

Highly nonspherical shapes of the fragments observed in these simulations, allow us to believe that all these features of the dynamical multifragmentation can be related to the negative surface tension coefficient in supercritical region.

In the developed model the contribution of surface tension induced by the repulsive interaction between the nuclear fragments is evaluated in a simplified form. Nevertheless, in combination with a finite incompressibility of liquid phase it gave us rather rich phase structure of the nuclear matter phase diagram. It is clear that a realistic short-range attraction between the nuclear fragments should also be included in order to correctly locate the region of the 2-nd order phase transition.

Acknowledgments. The authors appreciate the valuable comments of L. M. Satarov. V.V.S., A.I.I. and K.A.B. acknowledge a partial support of the Program 'On Perspective Fundamental Research in High Energy and Nuclear Physics' launched by the Section of Nuclear Physics of National Academy of Sciences of Ukraine. K.A.B. and I.N.M. acknowledge a partial support provided by the Helmholtz International Center for FAIR within the framework of the LOEWE program launched by the State of Hesse. The work of I.N.M. was also supported in part by the grant NSH-215.2012.2 (Russia).

\section{APPENDIX A}

In this Appendix we motivate for the parameterization of the liquid phase pressure (24). For this purpose, first, we consider a more general choice of the liquid phase pressure

$$
p_{L}=\frac{\tilde{W}+\mu-\mu_{0}+a_{\nu}\left[\mu-\mu_{0}\right]^{\nu}}{V_{1}}, \quad \text { for } \quad \nu=2,3,4,
$$


where $\tilde{W}(T)$ and $\mu_{0}$ are assumed to be the functions of $T$, i.e. $\tilde{W}(T)$ and $\mu_{0}(T)$. For the particle number $\rho_{L}$ and entropy $s_{L}$ densities of liquid phase one finds

$$
\begin{aligned}
& \rho_{L} \equiv \frac{\partial p_{L}}{\partial \mu}=\frac{1+a_{\nu} \nu \tilde{\mu}^{\nu-1}}{V_{1}}, \quad \text { with } \tilde{\mu} \equiv \mu-\mu_{0}, \\
& s_{L} \equiv \frac{\partial p_{L}}{\partial T}=\frac{1}{V_{1}}\left[\frac{d \tilde{W}}{d T}-\frac{d \tilde{\mu}_{0}}{d T} \frac{\rho_{L}}{\rho_{0}}\right] .
\end{aligned}
$$

In order to provide the nonnegative values of the liquid entropy density $s_{L} \geq 0$ at low and high particle densities $\rho_{L}$, it is necessary to require that $\mu_{0}=$ const. Then from (47) it is clearly seen that the liquid entropy density is positive for any large densities $\rho_{L} \gg \rho_{0}$, if $\mu_{0}=$ const and if $\frac{d \tilde{W}}{d T} \geq 0$. Moreover, according to the third law of thermodynamics, the entropy density of the system must vanish at $T=0$, i.e. $\left.\frac{d \tilde{W}}{d T}\right|_{T=0}=0$ which is fulfilled automatically, if $\tilde{W}(T)=W_{F m}(T)+C$, where $C$ is a constant to be found and $W_{F m}(T) \equiv \frac{T^{2}}{\varepsilon_{0}}\left(\varepsilon_{0}=16 \mathrm{MeV}\right)$ is the contribution of the excited states taken in the Fermi-gas approximation as in the original SMM [1].

In order to determine the constant $C$, consider the Gibbs criterion of phase equilibrium $\xi_{1}(T=$ $0, \mu)$ at $T=0$. Since at $T=0$ the gaseous pressure is zero, then an explicit form of such a criterions is as follows

$$
\tilde{W}(0)+\tilde{\mu}+a_{\nu} \tilde{\mu}^{\nu}=0 .
$$

From this algebraic equation for $\tilde{\mu}$ one deduces that for any real $\tilde{W}(0)$ value Eq. 48 has exactly $\nu$ algebraic roots. Analyzing Eqs. (46) and (48), one concludes that in order to have the nuclear liquid of normal nuclear density $\rho_{0}=V_{1}^{-1}$ at the phase equilibrium point $T=0$, it is necessary that $\tilde{\mu}=0$ is a solution of (48). Moreover, it is easy to see that this is the only physical solution. An existence of the solution $\tilde{\mu}=0$ of $(48)$ is provided by the condition $\tilde{W}(0)=0$. This condition along with the requirements for $\frac{d \tilde{W}}{d T}$ found above, unambiguously leads to that $\tilde{W}(T)=W_{F m}(T)=\frac{T^{2}}{\epsilon_{0}}$ and $C=0$, and, hence, $\mu_{0}=-W_{0}=-16 \mathrm{MeV}$. Such a choice not only obeys all the conditions discussed above, but also it automatically guarantees that in the vicinity of the normal nuclear state, i.e. at $T=0$ and $\rho_{L}=\rho_{0}$, the liquid phase of the present model (and its phase diagram) coincides with that one of the simplified SMM.

[1] J. P. Bondorf et al., Phys. Rep. 257, 131 (1995) and references therein.

[2] D. H. E. Gross, Phys. Rep. 279, 119 (1997).

[3] L. G. Moretto et al., Phys. Rep. 287, 249 (1997).

[4] P. Chomaz, M. Colonna and J. Randrup, Phys. Rep. 389, 263 (2004).

[5] K. A. Bugaev, Phys. Part. Nucl. 38, (2007), 447.

[6] A. S. Botvina and I. N. Mishustin, Nucl. Phys. A 843, 98 (2010).

[7] N. Buyukcizmeci et. al., Nucl. Phys. A 907, 13 (2013).

[8] N. Buyukcizmeci, A. S. Botvina and I. N. Mishustin, arXive:1304.6741 [nucl-th].

[9] I. N. Mishustin, Eur. Phys. J. A 30, 311 (2006).

[10] I. N. Mishustin, Phys. Rev. Lett. 82, 4779 (1999).

[11] G. Torrieri, B. Tomasik and I. N. Mishustin, Phys. Rev. C 77, 034903 (2008).

[12] S. Das Gupta and A.Z. Mekjian, Phys. Rev. C 57, 1361 (1998).

[13] S. Das Gupta, A. Majumder, S. Pratt, and A. Mekjian, nucl-th/9903007

[14] K. A. Bugaev, M. I. Gorenstein, I. N. Mishustin and W. Greiner, Phys. Rev. C62, 044320 (2000); arXiv:nucl-th/0007062 (2000).

[15] K. A. Bugaev, M. I. Gorenstein, I. N. Mishustin and W. Greiner, Phys. Lett. B 498, 144 (2001); arXiv:nucl-th/0103075 (2001).

[16] L. Beaulieu et al., Phys. Lett. B 463, 159 (1999).

[17] J. B. Elliott et al., (The EOS Collaboration), Phys. Rev. C 62, 064603 (2000).

[18] M. E. Fisher, Physics 3, 255 (1967).

[19] for a review on Fisher scaling see J. B. Elliott, K. A. Bugaev, L. G. Moretto and L. Phair, arXiv:nucl-ex/0608022 (2006) $36 \mathrm{p}$. and references therein.

[20] P. T. Reuter and K. A. Bugaev, Phys. Lett. B 517, 233 (2001).

[21] R. Ogul and A. S. Botvina, Phys. Rev. C 66, 051601 (2002). 
[22] V. A. Karnaukhov et al., Phys. Rev. C 67, 011601 R (2003).

[23] A. S. Botvina et al., Phys. Rev. C 74, 044609 (2006) and references therein.

[24] see, for instance, H. E. Stanley, Introduction to phase transitions and critical phenomena, Clarendon Press, Oxford, 1971.

[25] K. A. Bugaev, M. I. Gorenstein, H. Stöcker and W. Greiner, Phys. Lett. B 485, 121 (2000).

[26] G. Zeeb, K. A. Bugaev, P. T. Reuter and H. Stöcker, Ukr. J. Phys. 53, 279 (2008).

[27] K. A. Bugaev, Nucl. Phys. A 807, 251 (2008).

[28] K. A. Bugaev, Phys. Rev. C 76, 014903 (2007); Phys. Atom. Nucl. 71, 1615 (2008).

[29] K. A. Bugaev, V. K. Petrov and G. M. Zinovjev, Europhys. Lett. 85, 22002 (2009); Phys. Rev. C 79, 054913 (2009).

[30] A. Dillmann and G. E. Meier, J. Chem. Phys. 94, 3872 (1991).

[31] A. Laaksonen, I. J. Ford, and M. Kulmala, Phys. Rev. E 49, 5517 (1994).

[32] L. Van Hove, Physica 15, 951 (1949) ; Physica 16, 137 (1950).

[33] M. E. Fisher and B. U. Felderhof, Ann. of Phys. 58, 217 (1970).

[34] K. A. Bugaev, A. I. Ivanitskii, E. G. Nikonov, A. S. Sorin and G. M. Zinovjev, Can We Rigorously Define Phases in a Finite System?, Chapter 18 of the Proceedings of the XV-th Research Workshop "Nucleation Theory and Applications", held at JINR, Dubna, Russia, April 1- 30, 2011, edited by J. W. P. Schmelzer, G. Ropke, V. B. Priezzhev, Dubna JINR, 2011 ; arXiv:1106.5939 [nucl-th]

[35] K. A. Bugaev, V. K. Petrov and G. M. Zinovjev, Phys. Part. Nucl. Lett. 9, 238 (2012); arXiv:0904.4420 [hep-ph] (2009).

[36] K. A. Bugaev, L. Phair and J. B. Elliott, Phys. Rev. E 72, 047106 (2005).

[37] K. A. Bugaev and J. B. Elliott, Ukr. J. Phys. 52, 301 (2007).

[38] A. I. Ivanytskyi, K. A. Bugaev, A. S. Sorin and G. M. Zinovjev, Phys. Rev. E 86, 061107 (2012).

[39] L. M. Satarov, M. N. Dmitriev and I. N. Mishustin, Phys. Atom. Nucl. 72, 1390 (2009).

[40] K. A. Bugaev et al., Phys. Atom. Nucl. 75, 707 (2012); arXiv:1101.4549 [hep-ph]

[41] K. A. Bugaev, Acta. Phys. Polon. B 36, 3083 (2005).

[42] D. Vretenar, T. Niksic, and P. Ring, Phys. Rev. C 68, 024310 (2003).

[43] G. Colo and Nguyen Van Giai, Nucl. Phys. A 731, 15 (2004).

[44] E. Khan, Phys. Rev. C 80, 011307(R) (2009).

[45] V. B. Soubbotin, V. I. Tselyaev and X. Vinas, Phys. Rev. C 69, 064312 (2004).

[46] N. Buyukcizmeci et al., arXiv:1211.5990 2 [nucl-th].

[47] X. Campi, H. Krivine E. Plagnol and N. Sator, Phys. Rev. C 67, 044610 (2003). 


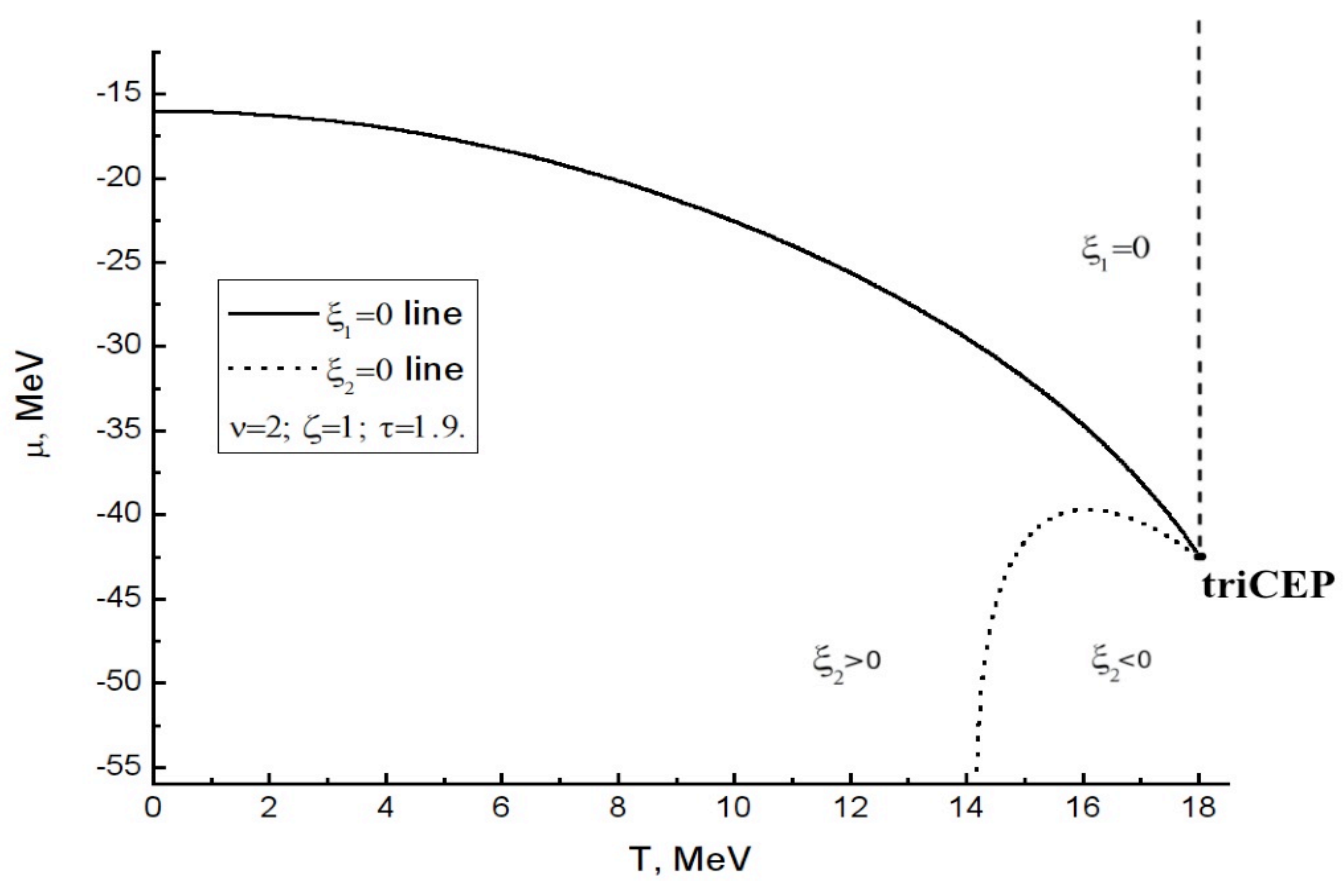

FIG. 1: Phase diagram in $T-\mu$ plane for the case $\tau=1.9, \nu=2$. At the critical temperature $T_{\text {cep }}=18 \mathrm{MeV}$ there is a triCEP. The solid curve shows a 1-st order PT, the long dashed one shows a 2-nd order PT, while the short dashed curve indicates the nil line of the surface tension coefficient. 


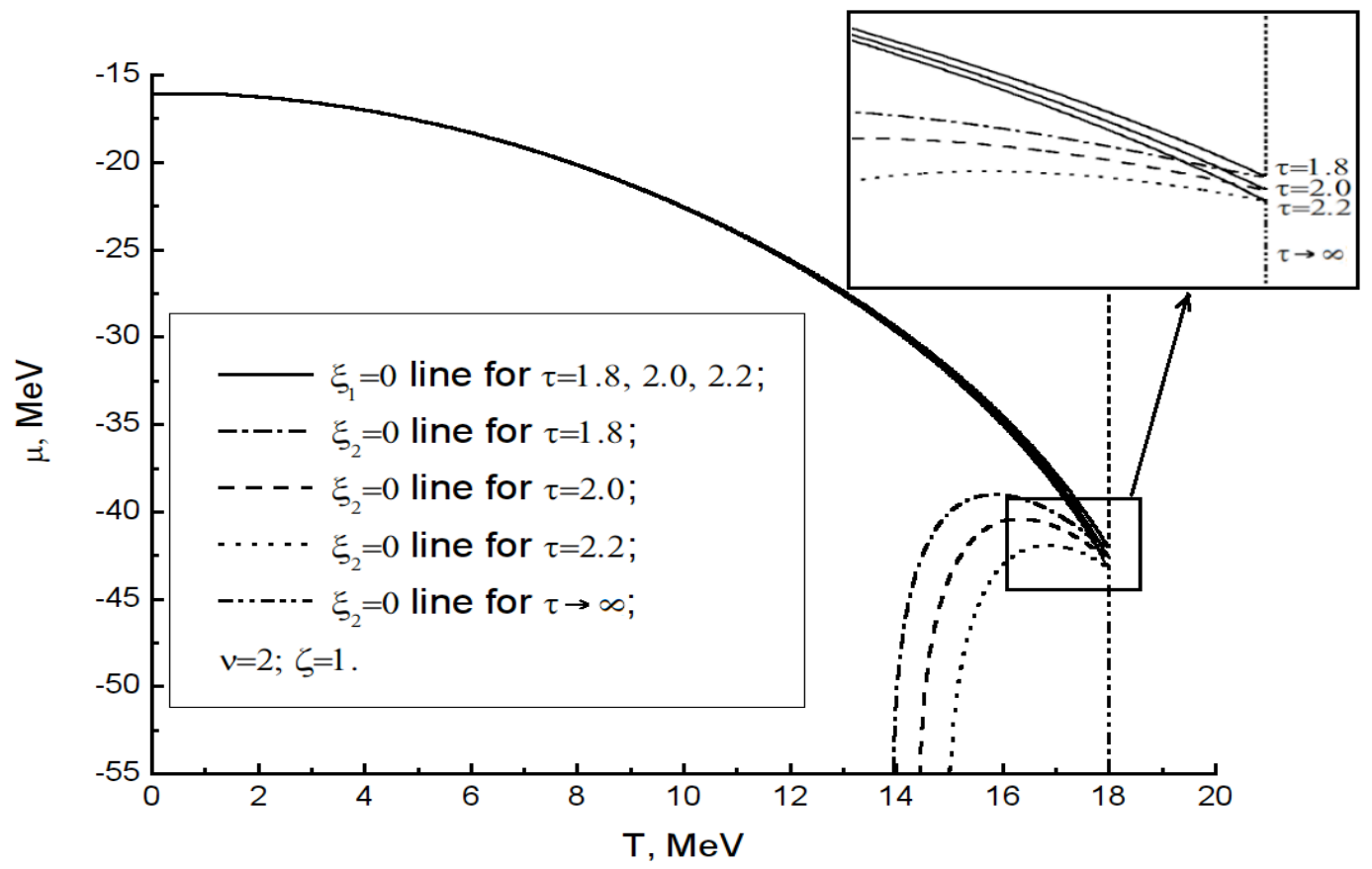

FIG. 2: Phase diagram in $T-\mu$ plane for $\nu=2$ is shown for several values of the Fisher topological parameter $\tau$. The line of the 2-nd order PT (vertical short dashed line) is shown for $\tau=1.8$ only, since other lines are hardly distinguishable from each other. Also the nil surface tension line of the limiting case $\tau \rightarrow \infty$ is shown for a comparison by the vertical dashed-double-dotted line.

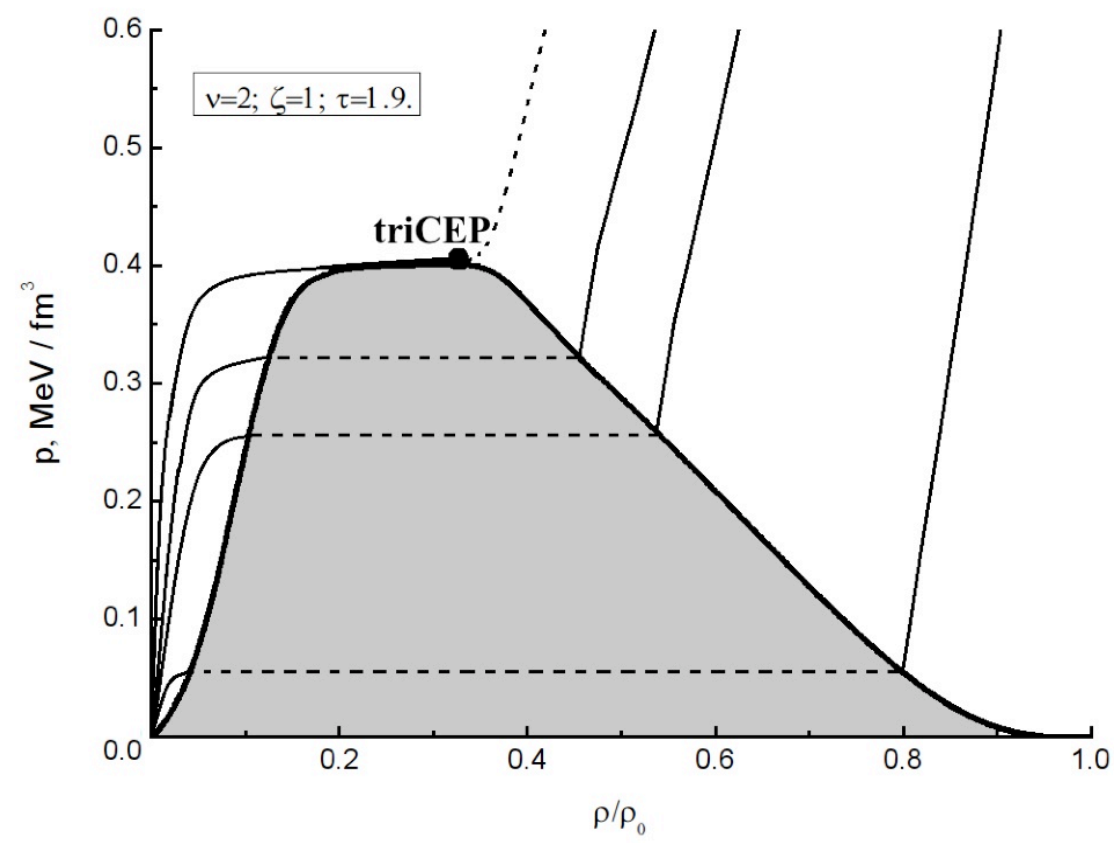

FIG. 3: Phase diagram in $\rho-p$ plane for $\nu=2$ and $\tau=1.9$. The grey area corresponds to a mixed phase of the 1-st order PT. The isotherms are shown for $\mathrm{T}=11,16,17,18 \mathrm{MeV}$ from bottom to top. For the density $\rho / \rho_{0} \geq 1 / 3$ at the isotherm $T=18$ $\mathrm{MeV}$ there exists the 2-nd order PT (dashed curve). 


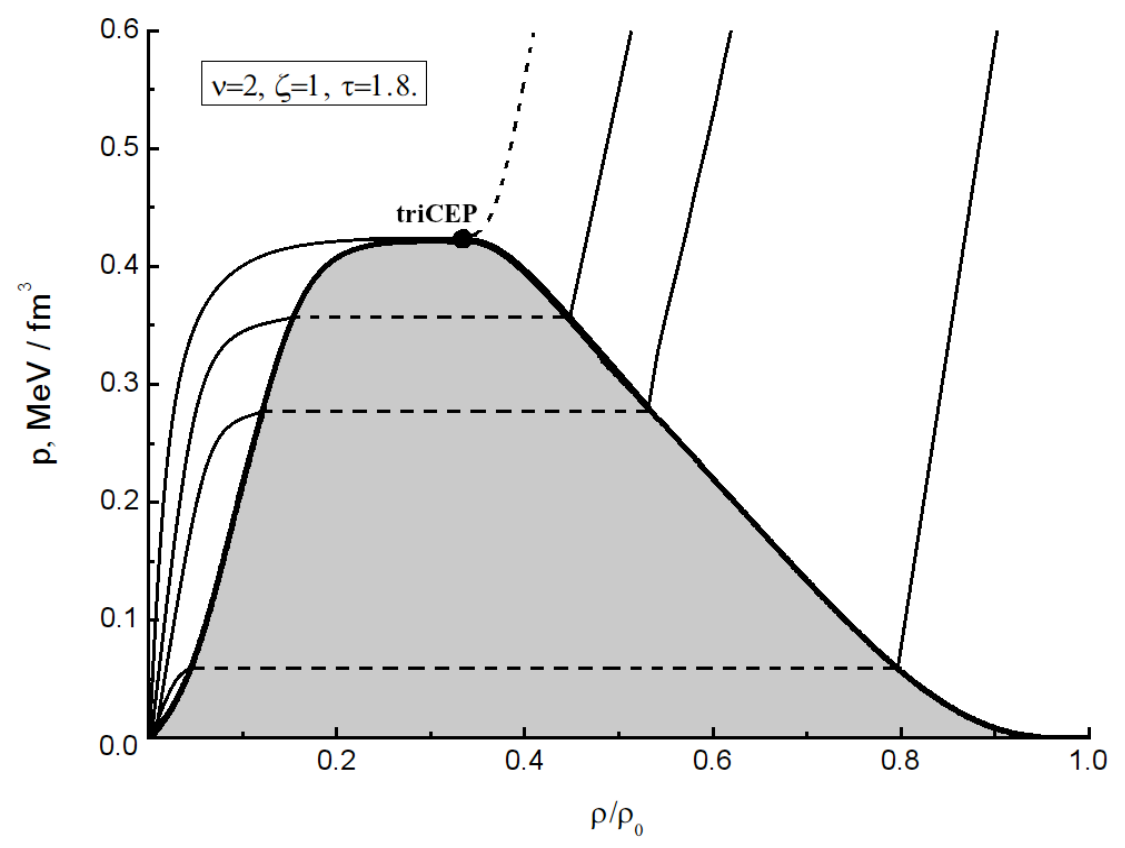

FIG. 4: The same as in Fig. 3 but for the Fisher parameter $\tau=1.8$.

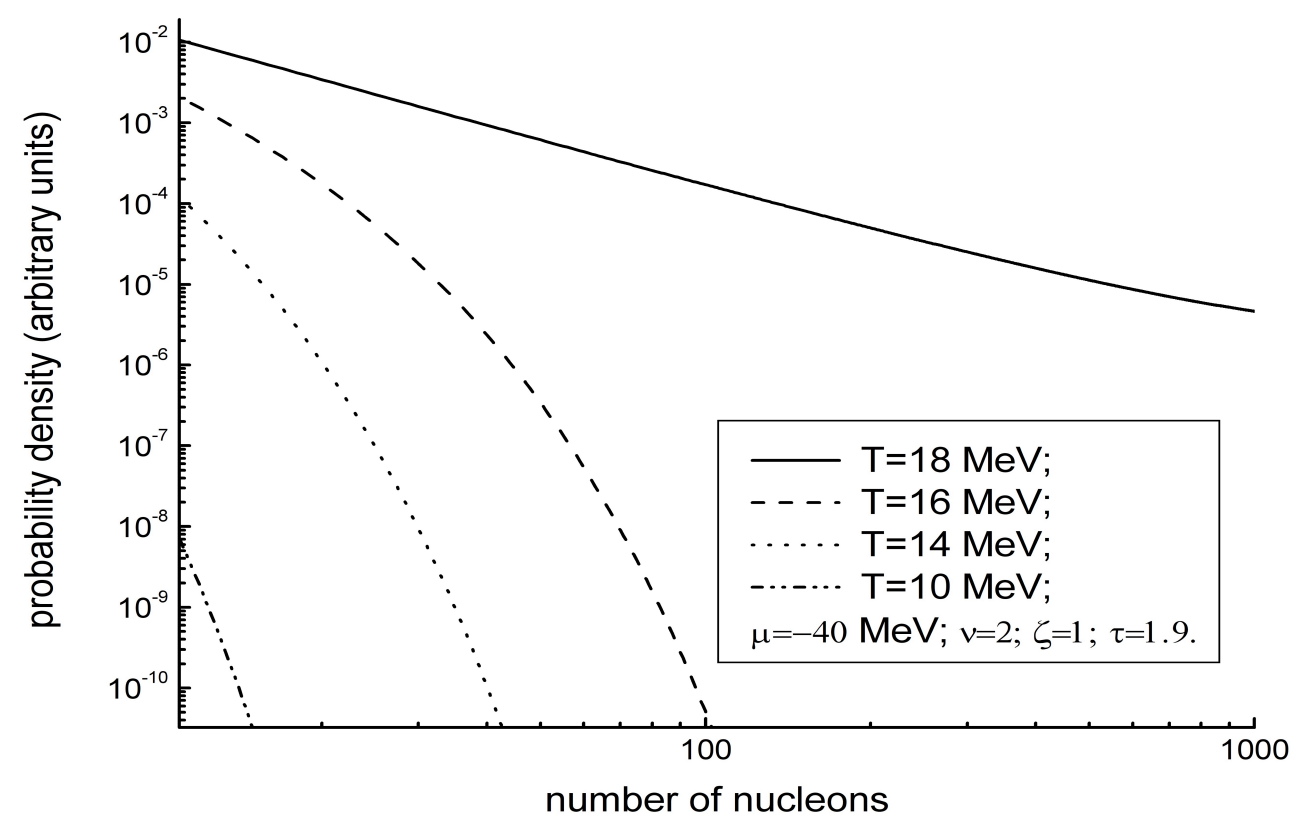

FIG. 5: Fragment size distribution in the gaseous phase is shown for different temperatures and fixed baryonic chemical potential and $\nu=2$. For all temperatures below $T_{c e p}=18 \mathrm{MeV}$ the surface tension coefficient is positive, while it vanishes for $T=T_{c e p}$. As temperature increases the distribution changes from the exponential one to a power law which is a straight line in a double logarithmic scale. 


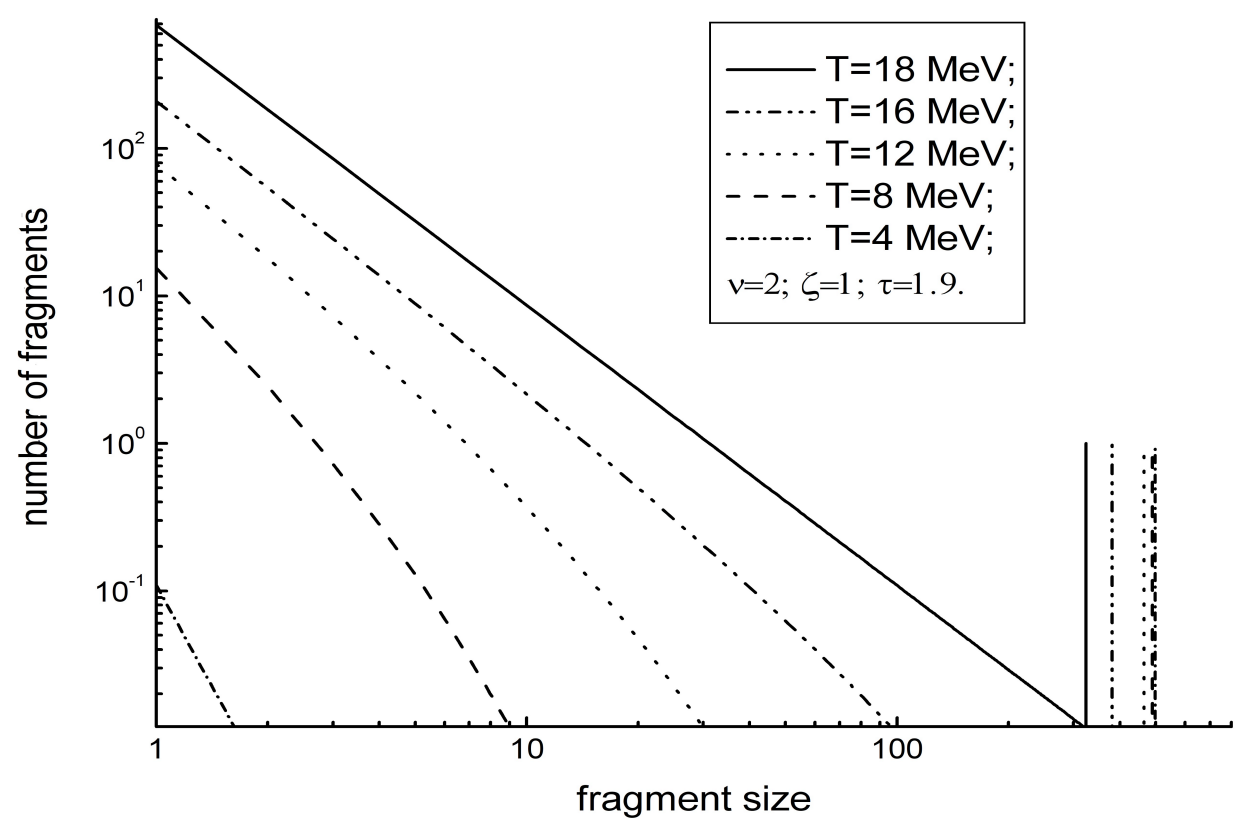

FIG. 6: Fragment size distribution in the mixed phase is shown for different temperatures and fixed particle number density $\rho=\frac{\rho_{0}}{3}$ and $\nu=2$. For all temperatures below $T_{c e p}=18 \mathrm{MeV}$ the surface tension coefficient is positive, while it vanishes for $T^{3}=T_{\text {cep }}$. All these distributions were normalized for 500 nucleons in the system. The liquid cluster is represented by a Kronecker delta function. As temperature increases the distribution function of the gas changes from the exponential one to a power law which is a straight line in a double logarithmic scale.

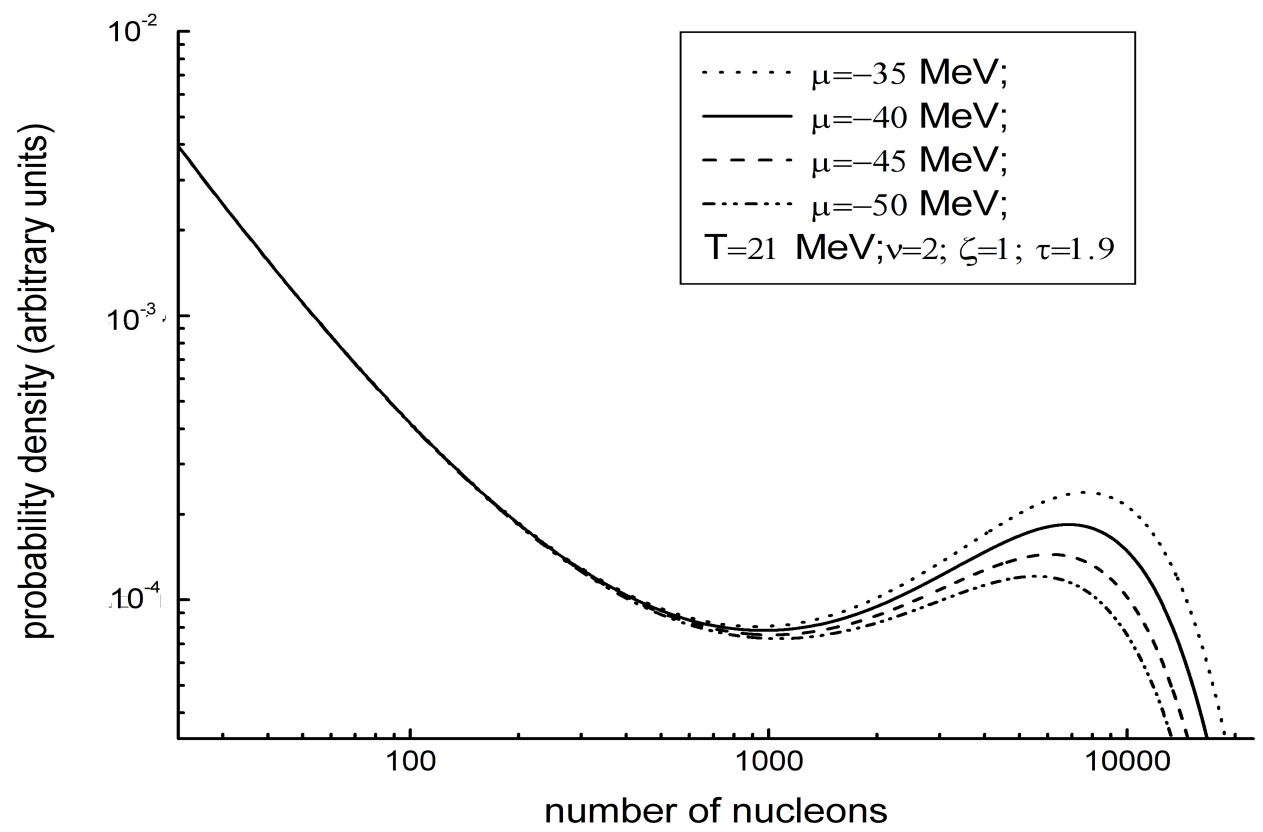

FIG. 7: Fragment size distribution in the phase with the negative value of the surface tension coefficient of the model with $\nu=2$ is shown for a fixed temperature and different baryonic chemical potentials. As baryonic chemical potential increases the maximum of the distribution function grows higher and wider, and it shifts towards the larger number of nucleons in a fragment. 


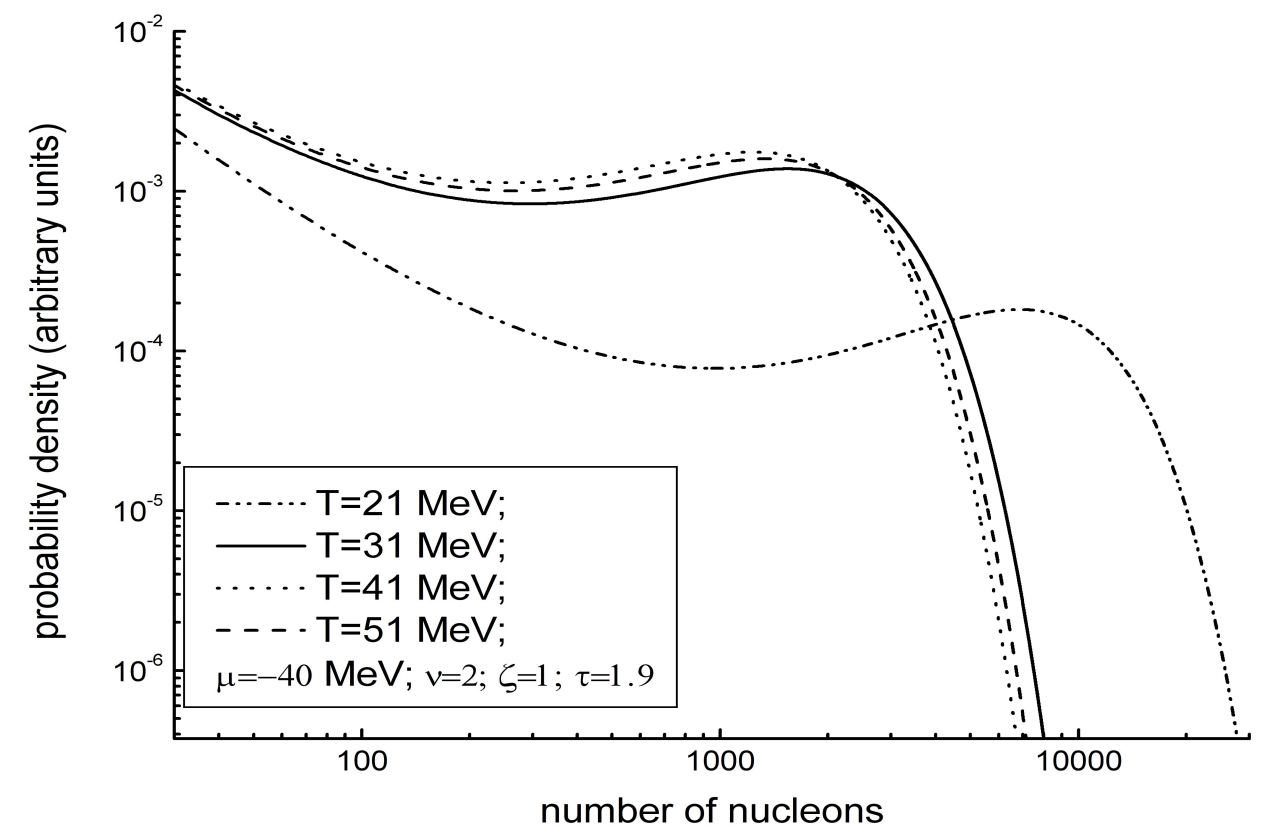

FIG. 8: Fragment size distribution in the phase with the negative value of the surface tension coefficient of the model with $\nu=2$ is shown for a fixed baryonic chemical potential and different temperatures. As temperature increases the minimum and maximum of the distribution function grow wider and shallower and they shift towards the smaller number of nucleons in a fragment. 